\title{
Stratification Effects in the Turbulent Boundary Layer beneath a Melting Ice Shelf: Insights from Resolved Large-Eddy Simulations
}

\author{
CATHERIne A. VReugdenhil AND JOHn R. TAYLOR \\ University of Cambridge, Cambridge, United Kingdom
}

(Manuscript received 26 November 2018, in final form 15 March 2019)

\begin{abstract}
Ocean turbulence contributes to the basal melting and dissolution of ice shelves by transporting heat and salt toward the ice. The meltwater causes a stable salinity stratification to form beneath the ice that suppresses turbulence. Here we use large-eddy simulations motivated by the ice shelf-ocean boundary layer (ISOBL) to examine the inherently linked processes of turbulence and stratification, and their influence on the melt rate. Our rectangular domain is bounded from above by the ice base where a dynamic melt condition is imposed. By varying the speed of the flow and the ambient temperature, we identify a fully turbulent, well-mixed regime and an intermittently turbulent, strongly stratified regime. The transition between regimes can be characterized by comparing the Obukhov length, which provides a measure of the distance away from the ice base where stratification begins to dominate the flow, to the viscous length scale of the interfacial sublayer. Upper limits on simulated turbulent transfer coefficients are used to predict the transition from fully to intermittently turbulent flow. The predicted melt rate is sensitive to the choice of the heat and salt transfer coefficients and the drag coefficient. For example, when coefficients characteristic of fully developed turbulence are applied to intermittent flow, the parameterized three-equation model overestimates the basal melt rate by almost a factor of 10 . These insights may help to guide when existing parameterizations of ice melt are appropriate for use in regional or large-scale ocean models, and may also have implications for other iceocean interactions such as fast ice or drifting ice.
\end{abstract}

\section{Introduction}

Ocean-driven melting of ice shelves around Antarctica has the potential to play an important role in accelerating sea level rise (Jacobs et al. 2002; Rignot and Jacobs 2002; Rye et al. 2014; Harig and Simons 2015). Ice shelves are the floating extensions of ice sheets that act to buttress land-bounded ice and prevent it sliding into the ocean. The thinning of ice shelves can reduce the resistance to the flow of ice upstream (Schoof 2007; Gudmundsson 2013) or melt basal channel cavities that weaken the entire shelf (Rignot and Steffen 2008; Alley et al. 2016), resulting in calving events and land ice moving into the ocean, thereby raising the sea level. The regions near Antarctic ice shelves are also important for the modification of water masses, such as in the formation of the densest water mass in the ocean (Antarctic Bottom Water), which feeds the downwelling limb of the global meridional overturning circulation (Nicholls et al. 2009;

Corresponding author: Catherine A. Vreugdenhil, c.a.vreugdenhil@ damtp.cam.ac.uk
Purkey and Johnson 2012). Changes in the interaction between ice sheets and the ocean could affect the dense water formation rate and influence the global transport of heat and hence the climate (Snow et al. 2018). Key to predicting future climate scenarios is understanding the processes governing the ice shelf melt rates and response to changes in ocean circulation.

Observations of ice shelf melt and the underlying ocean circulation show contrasting behavior at different locations around Antarctica. Data taken by drilling through the Larsen $\mathrm{C}$ ice shelf on the Antarctic peninsula show well-mixed profiles of temperature and salinity up to 20-30 m beneath the basal surface with an underlying weakly stable stratification, a high current speed, and a strong tidal signal (Nicholls et al. 2012). The temperature difference between a few meters depth and the iceocean interface, known as the thermal driving, is small $\left(\Delta T=0.08^{\circ} \mathrm{C}\right)$ and the basal melt rate is modest at $1.9 \mathrm{~m} \mathrm{yr}^{-1}$. This picture of energetic flow with a weak stratification has also been observed beneath the Ronne ice shelf (Jenkins et al. 2010), Fimbul ice shelf (Hattermann et al. 2012), and Ross ice shelf (Arzeno et al. 2014). 
In contrast, the water column beneath the George VI ice shelf is highly stratified with a low current speed and a weak tidal signature (Kimura et al. 2015). Here, the thermal driving is large $\left(\Delta T=2.3^{\circ} \mathrm{C}\right)$ but the melt rate, measured using upward-looking sonar, remains modest at $1.4 \mathrm{~m} \mathrm{yr}^{-1}$ (Kimura et al. 2015). Borehole measurements near the grounding line of the Ross ice shelf also show strong stratification in quiescent flow and low melt rates (Begeman et al. 2018). Other strongly stratified layers have been observed beneath the Pine Island Glacier ice shelf, where data from an autonomous underwater vehicle (AUV) show a sharp temperature gradient maintained close to the ice shelf and a slow horizontal current speed (Kimura et al. 2016). In a different area under the Pine Island Glacier ice shelf, borehole measurements also show a stratified boundary layer, but here the flow is dominated by melt-generated buoyancy acting on the sloping base of the ice shelf (Stanton et al. 2013). The extreme Antarctic environment means that observations are sparse and lack the resolution to fully characterize the processes controlling the melt rate when the oceanic boundary layer is turbulent compared to when it is more strongly stratified.

The structure of the ocean boundary layer beneath the ice is often characterized by an interfacial sublayer (on the order of millimeters to centimeters) where molecular viscosity or roughness dominates the flow, followed by a surface layer (a few meters) where the logarithmic "law of the wall" scaling applies, and finally an outer planetary boundary layer (tens of meters) where Earth's rotation limits the mixing length (Holland and Jenkins 1999; McPhee 2008). If the flow is strongly stratified, the law-of-the-wall scaling will not hold in the surface layer and stratification will limit the maximum mixing length in the outer layer. In cases of very strong stratification and weak shear, the dynamics may be dominated by free convection (Martin and Kauffman 1977; Keitzl et al. 2016) or double-diffusive layers, the latter of which is theorized to apply to regions of the ocean boundary layer below the George VI (Kimura et al. 2015) and Ross (Begeman et al. 2018) ice shelves. The picture becomes more complicated when there is a buoyancy-driven plume adjacent to the ice, which can occur when the ice is significantly sloped such as near the grounding line, and entrainment into the plume determines the heat transferred to the ice and hence the melt rate (Jenkins 2016; McConnochie and Kerr 2018). Here, we focus on the ISOBL without a significant slope to be consistent with ice shelf observations further from the grounding line (e.g., Nicholls et al. 2012; Kimura et al. 2015).

In most ocean models, computational limitations mean that the ISOBL cannot be fully resolved and must be parameterized to achieve a realistic melt rate. There are a wide range of parameterizations, but none completely capture the dynamics of the ocean boundary layer and its response to the melt rate. One common parameterization, known as the three-equation model, is based on the relatively simple concept of parameterizing the turbulent fluxes of heat and salt into transfer coefficients (Holland and Jenkins 1999; McPhee 2008). Comparing the parameterization against observations, the three-equation model works reasonably well in some locations such as the Ronne ice shelf (Jenkins et al. 2010). However, the three-equation model does not work in other locations such as the George VI ice shelf where it overestimates the true melt rate by more than an order of magnitude (Kimura et al. 2015). This is likely because the influence of stratification on turbulence is not included in this parameterization. The three-equation model is also known to poorly estimate the melt rate in regions where the ice is significantly sloped and there is a buoyancy-driven plume (McConnochie and Kerr 2017).

Monin-Obukhov similarity theory was formulated to describe the influence of stratification effects on a turbulent boundary layer (Monin and Obukhov 1954). The Obukhov length is a measure of the distance away from the ice where stratification starts to dominate the flow (Obukhov 1946). Here, building on previous work (McPhee 2008; Deusebio et al. 2015; Scotti and White 2016; Zhou et al. 2017), we find that the Obukhov length provides a useful way to characterize the influence of density stratification on turbulence in the law-of-the-wall region of the ISOBL. Large values of the Obukhov length imply that stratification does not affect the near-ice flow, while small values imply that more of the ISOBL is susceptible to stratification effects. If the Obukhov length is comparable to the viscous sublayer thickness, then there is no region of the flow free of either viscous or stratification effects, both of which damp out turbulence (Pope 2000; Flores and Riley 2011). In this case the law-of-thewall scaling is not expected to hold and the flow is susceptible to laminarization. The ratio of the Obukhov length to the thickness of the viscous sublayer has been used to describe the transition between turbulent, intermittent, and laminar flow in a stable atmospheric boundary layer (Flores and Riley 2011) and stratified plane Couette flow (Deusebio et al. 2015; Zhou et al. 2017).

The present study is motivated by ocean-driven melting beneath ice shelves. We use large-eddy simulations (LES) with a state-of-the-art turbulent parameterization (Rozema et al. 2015; Abkar et al. 2016) to examine steady, unidirectional flow with an unstratified free stream as a model of a small region near the ice. As outlined in section 2 the model is designed to resolve the viscous sublayer and surface layer, only parameterizing the smallest scales of turbulence. Our focus is on turbulence 
very near the ice. Our computational domain can be viewed as a small region embedded within the deeper planetary boundary, so for simplicity we do not include Earth's rotation. The majority of simulations use a flat ice base, perpendicular to the direction of gravity. Scaling theory for the viscous sublayer and surface layer is outlined in section 3, along with the three-equation parameterization. The results in section 4 explore different far-field currents that generate shear turbulence, and a range of imposed far-field temperatures. The focus is on understanding the influence of stratification associated with the input of meltwater on turbulence and the subsequent feedbacks on the melt rate. A summary of the results is in section 5. In section 6 we discuss the applicability of our results to the ocean. While the motivation for this study was the ice shelf-ocean boundary layer, the simulations are idealized enough that they also have implications for other applications, including the boundary layer beneath sea ice.

\section{Model design}

Here, we model the ocean boundary layer under an ice shelf in a rectangular domain of length $L_{x}$, width $L_{y}$, and height $h$ (Fig. 1). The flow is bounded from above by the base of the ice shelf which is assumed to be flat. The upper and lower boundaries are impenetrable, while the two horizontal directions are periodic. A no-slip condition is imposed on the upper boundary (the ice base) and a free-slip condition on the lower boundary. For most of the simulations, we assume that the ice shelf is horizontal with gravity perpendicular to the ice-ocean interface and no rotation term. Simulations with small basal slope angles are discussed in appendix A and give very similar results to the simulations with a flat ice base.

The simulations solve the incompressible, nonhydrostatic Navier-Stokes momentum equation under the Boussinesq approximation along with the conservation of mass, heat, and salt, and a linear equation of state, respectively:

$$
\begin{aligned}
\frac{D \mathbf{u}}{D t} & =-\frac{1}{\rho_{0}} \nabla p+\nu \nabla^{2} \mathbf{u}+F \mathbf{i}+\frac{\Delta \rho}{\rho_{0}} g \mathbf{k}-\nabla \cdot \boldsymbol{\tau}, \\
\nabla \cdot \mathbf{u} & =0, \\
\frac{D T}{D t} & =\kappa_{T} \nabla^{2} T+R_{T}-\nabla \cdot \lambda_{T}, \\
\frac{D S}{D t} & =\kappa_{S} \nabla^{2} S+R_{S}-\nabla \cdot \lambda_{S}, \quad \text { and } \\
\frac{\Delta \rho}{\rho_{0}} & =-\alpha\left(T-T_{0}\right)+\beta\left(S-S_{0}\right),
\end{aligned}
$$

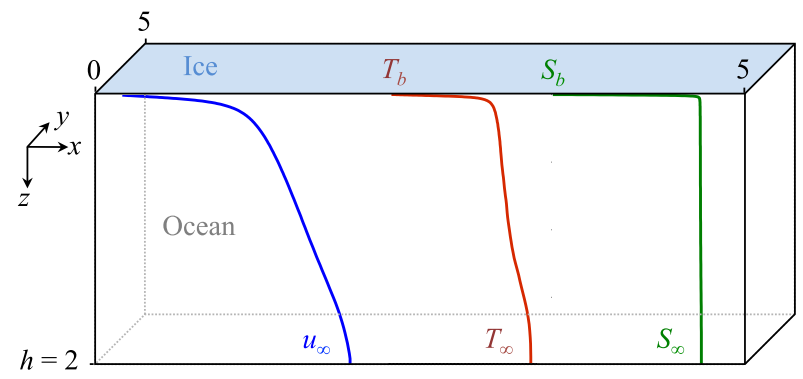

FIG. 1. Setup of the numerical simulations which model the upper region of the ocean boundary layer beneath an ice shelf. Vertical profiles of velocity (blue curve), temperature (red), and salinity (green) have been horizontally averaged across the domain. The profiles are from a run with friction velocity $u_{*}=$ $0.05 \mathrm{~cm} \mathrm{~s}^{-1}$, far-field temperature $T_{\infty}=-2.18^{\circ} \mathrm{C}$ and salinity $S_{\infty}=35$ psu (run 6 in Table 1 ). The resulting thermal driving is relatively weak $\Delta T=0.0031^{\circ} \mathrm{C}$. Note that the vertical $z$ direction is defined as positive downward, and domain sizes are in meters.

where $\mathbf{u}=(u, v, w)$ is the velocity vector, $(x, y, z)$ is the position vector, $t$ is time, $p$ is pressure, $T$ is temperature, $S$ is salinity, $\Delta \rho=\rho-\rho_{0}$ is the departure of density $\rho$ from the reference value $\rho_{0}, T_{0}$ is the reference temperature, and $S_{0}$ reference salinity, $g=$ $9.81 \mathrm{~m} \mathrm{~s}^{-2}$ is the gravitational acceleration, $\mathbf{i}$ and $\mathbf{k}$ are the unit vectors in the $x$ and $z$ directions, and $\alpha=3.87 \times 10^{-5}{ }^{\circ} \mathrm{C}^{-1}$ and $\beta=7.86 \times 10^{-4} \mathrm{psu}^{-1}$ are the coefficients of thermal expansion and saline contraction, respectively (Jenkins 2011). We use realistic values of the molecular viscosity $\nu=1.8 \times 10^{-6} \mathrm{~m}^{2} \mathrm{~s}^{-1}$ and the molecular diffusivity of heat $\kappa_{T}=1.3 \times 10^{-7} \mathrm{~m}^{2} \mathrm{~s}^{-1}$ (Prandtl number $\left.\operatorname{Pr}=\nu / \kappa_{T}=14\right)$ and salt $\kappa_{S}=7.2 \times 10^{-10} \mathrm{~m}^{2} \mathrm{~s}^{-1}$ (Schmidt number Sc $\left.=\nu / \kappa_{S}=2500\right)$.

A far-field current is produced by imposing a mean pressure gradient in the $x$ direction. In Eq. (1) this constant driving force appears as $F=-\left(1 / \rho_{0}\right) \partial p / \partial x$, where $p$ is the mean pressure. In an equilibrated state the net momentum input by the pressure gradient must be balanced by the wall shear stress $\tau_{b}=\rho_{0} \nu|\partial u / \partial z|_{b}$,

$$
-\int_{0}^{h} \frac{\partial \underline{p}}{\partial x} d z=\tau_{b}
$$

where the subscript $b$ refers to the ice-ocean boundary. By imposing a pressure gradient, we are effectively setting the wall shear stress and hence the friction velocity $u_{*}=\sqrt{\tau_{b} / \rho_{0}}$ in equilibrated state. Two values of the pressure gradient are chosen to produce equilibrated state friction velocities of $u_{*}=0.05 \mathrm{~cm} \mathrm{~s}^{-1}$ and $u_{*}=$ $0.1 \mathrm{~cm} \mathrm{~s}^{-1}$, which result in far-field velocities of $u_{\infty}=1-$ $9 \mathrm{~cm} \mathrm{~s}^{-1}$. Here, the far-field means the maximum depth in the domain of $z=h$ and is indicated by the subscript $\infty$.

To maintain the far-field temperature and salinity, the lower quarter of the domain is relaxed on a time scale of 
$\tau$ to chosen far-field temperature $T_{\infty}$ and salinity $S_{\infty}$ values. In the heat [Eq. (3)] and salt [Eq. (4)] conservation equations, the relaxation terms are

$$
\begin{aligned}
& R_{T}=-\frac{1}{\tau}\left(\langle T\rangle-T_{\infty}\right) e^{-\left[C_{f}(h-z) / h\right]^{2}}, \quad \text { and } \\
& R_{S}=-\frac{1}{\tau}\left(\langle S\rangle-S_{\infty}\right) e^{-\left[C_{f}(h-z) / h\right]^{2}},
\end{aligned}
$$

respectively, where the angle brackets imply a horizontal average and the stretching factor $C_{f}=7$. The relaxation time scale is based on a far-field velocity of $u_{\infty} \sim 1 \mathrm{~cm} \mathrm{~s}^{-1}$ such that $\tau=h / u_{\infty} \sim 200$ s on the basis that eddies will mix the scalar fields on a similar time scale.

The governing equations [Eqs. (1)-(5)] are discretized using Fourier modes in the two horizontal directions and second-order finite differences in the vertical direction (see Taylor 2008). Note that Eqs. (1)-(5) are the grid-filtered equations where $\mathbf{u}, T$, and $S$ are the resolved fields. A recently developed LES parameterization known as the anisotropic minimum dissipation model (Rozema et al. 2015; Vreugdenhil and Taylor 2018) is used to evaluate the subfilter stress tensor $\boldsymbol{\tau}$ and subfilter scalar fluxes of heat $\lambda_{T}$ and salt $\lambda_{S}$ (see appendix B for more details). The time-stepping uses a low-storage third-order Runge-Kutta method for the nonlinear terms and a semi-implicit CrankNicholson method for the viscous and diffusive terms. A $2 / 3$ dealiasing rule is applied moving from Fourier back to physical space (Orszag 1971).

A no-flux boundary condition is applied to the temperature and salinity at the lower boundary and a melting ice condition at the upper ice-ocean boundary. The volume input of water due to ice melting is expected to be very small compared to the current velocity, hence we assume zero volume input (Holland and Jenkins 1999). The salinity of ice and the conduction of heat through the ice are also assumed to be zero. Very low, near-zero salinities are typically observed in ice shelves (Oerter et al. 1992; Eicken et al. 1994). The condition of no heat conducted through the ice shelf has been used regularly in past studies on the assumption that the conducted heat flux is small compared to the latent heat flux (Determann and Gerdes 1994; Jenkins and Bombosch 1995; Grosfeld et al. 1997; Williams et al. 1998; Holland and Jenkins 1999; Gayen et al. 2016; Mondal et al. 2019). The resulting equations at the ice-ocean boundary are the conservation of heat and salt, along with the liquidus condition,

$$
\begin{aligned}
c_{w} \rho_{w} \kappa_{T} \frac{\partial T}{\partial z} & =\rho_{i} L_{i} m, \\
\rho_{w} \kappa_{S} \frac{\partial S}{\partial z} & =\rho_{i} S_{b} m, \text { and }
\end{aligned}
$$

$$
T_{b}=\lambda_{1} S_{b}+\lambda_{2}+\lambda_{3} P
$$

which are solved for the melt rate $m$, temperature $T_{b}$, and salinity $S_{b}$ at the ice-ocean boundary (see appendix $\mathrm{C}$ for numerical method) following similar methods to Gayen et al. (2016). The subscript $w$ refers to parameters corresponding to water and subscript $i$ to parameters corresponding to ice. The specific heat capacity of water is $c_{w}=3974 \mathrm{~J} \mathrm{~kg} \mathrm{~g}^{-1}{ }^{\circ} \mathrm{C}^{-1}$, the latent heat of fusion is $L_{i}=3.35 \times 10^{5} \mathrm{~J} \mathrm{~kg}^{-1}$, and $\lambda_{1}=-5.73 \times 10^{-2}{ }^{\circ} \mathrm{C}, \lambda_{2}=$ $8.32 \times 10^{-2}{ }^{\circ} \mathrm{C}$, and $\lambda_{3}=-7.53 \times 10^{-4}{ }^{\circ} \mathrm{C} \mathrm{dbar}^{-1}$ are coefficients in a linearized expression for the freezing point of seawater (Jenkins 2011). The locally hydrostatic background pressure due to the depth of the ice base below sea level $P=350 \mathrm{dbar}$ is chosen to be broadly consistent with the Larsen C ice shelf (Nicholls et al. 2012).

The domain size for all runs was set to $L_{x} \times L_{y} \times h=$ $5 \mathrm{~m} \times 5 \mathrm{~m} \times 2 \mathrm{~m}$. The computational grid for the $u_{*}=0.05 \mathrm{~cm} \mathrm{~s}^{-1}$ case was $128 \times 128 \times 145$ and for the $u_{*}=0.1 \mathrm{~cm} \mathrm{~s}^{-1}$ case was $256 \times 256 \times 289$. These grids were chosen to be consistent with the criteria outlined in Vreugdenhil and Taylor (2018) for resolved LES. One exception was that a $1 / 8$ vertical-to-horizontal grid cell aspect ratio at the edge of the viscous layer was found to work just as well as a 1/4 aspect ratio, thus the former was chosen to allow more grid stretching in the vertical direction. The vertical grid was stretched to place more grid cells adjacent to the ice to resolve the near-ice conductive and diffusive sublayers, which are thin because of the realistic values of $\kappa_{T}$ and $\kappa_{S}$. The grid stretching function is $z_{k}=h-$ $h \tanh \left[S_{f}(k-1) / N_{z}\right] / \tanh \left(S_{f}\right)$, where $k$ is the grid cell number, $N_{z}$ is the total number of grid cells, and $S_{f}=3.5$ is the grid stretching. This resulted in $\Delta z_{\min }=0.019 \mathrm{~cm}$, $\Delta z_{\max }=4.9 \mathrm{~cm}$ for the $u_{*}=0.05 \mathrm{~cm} \mathrm{~s}^{-1}$ cases and $\Delta z_{\min }=$ $0.009 \mathrm{~cm}, \Delta z_{\max }=2.5 \mathrm{~cm}$ for the $u_{*}=0.1 \mathrm{~cm} \mathrm{~s}^{-1}$ cases.

A range of far-field temperatures $T_{\infty}$ are chosen to achieve thermal driving of $\Delta T=0.005^{\circ}-0.43^{\circ} \mathrm{C}$ (Table 1). The far-field salinity was set to $S_{\infty}=35$ psu for all cases. Additional passive scalar runs were conducted at each friction velocity by setting the gravity term in Eq. (1) to zero $(g=0)$. These runs were designed to examine the transport of heat and salt when the scalars do not influence the flow. The simulations were run with chosen values of $T_{\infty}$ to result in $\Delta T$ and a particular melt rate. However, as outlined in section 4 , in the passive scalar case the melt rate is dependent only on $\Delta T$ (for a particular $u_{*}$ and $S_{\infty}$ ) and so the chosen value of $T_{\infty}$ is arbitrary. Hence values of $T_{\infty}, \Delta T$ and the melt rate have not been included for the passive scalar cases in Table 1 because the runs apply more generally.

Each melting scenario is initialized from an equilibrated fully turbulent flow, with uniform temperature and salinity profiles set to the chosen far-field values $T_{\infty}$ 
TABLE 1. Run summary varying friction velocity $u_{*}$ (set by imposing a chosen pressure gradient) and far-field temperature $T_{\infty}$. Results are the time-averaged measured friction velocity $u_{*}$, thermal driving $\Delta T=T_{\infty}-T_{b}$, melt rate, drag coefficient $C_{d}$, transfer coefficients for heat $\Gamma_{T}$ and salt $\Gamma_{S}$, and Obukhov length scale ratio $L^{+}$. Runs 9 and 16 have $g=0$.

\begin{tabular}{|c|c|c|c|c|c|c|c|c|c|}
\hline Run & $u_{*} \operatorname{set}\left(\mathrm{cm} \mathrm{s}^{-1}\right)$ & $T_{\infty}\left({ }^{\circ} \mathrm{C}\right)$ & $u_{*}$ measured $\left(\mathrm{cm} \mathrm{s}^{-1}\right)$ & $\Delta T\left({ }^{\circ} \mathrm{C}\right)$ & Melt rate $\left(\mathrm{m} \mathrm{yr}^{-1}\right)$ & $C_{d}$ & $\Gamma_{T}$ & $\Gamma_{S}$ & $L^{+}$ \\
\hline 1 & 0.05 & -2.00 & 0.0451 & 0.1405 & 0.0357 & $7.75 \times 10^{-5}$ & $1.47 \times 10^{-3}$ & $1.05 \times 10^{-4}$ & 214 \\
\hline 2 & 0.05 & -2.07 & 0.0457 & 0.0857 & 0.0331 & $8.43 \times 10^{-5}$ & $2.19 \times 10^{-3}$ & $1.45 \times 10^{-4}$ & 225 \\
\hline 3 & 0.05 & -2.10 & 0.0469 & 0.0641 & 0.0408 & $1.49 \times 10^{-4}$ & $3.53 \times 10^{-3}$ & $2.41 \times 10^{-4}$ & 268 \\
\hline 4 & 0.05 & -2.15 & 0.0499 & 0.0242 & 0.0214 & $3.68 \times 10^{-4}$ & $4.60 \times 10^{-3}$ & $2.23 \times 10^{-4}$ & 486 \\
\hline 5 & 0.05 & -2.17 & 0.0497 & 0.0093 & 0.0116 & $7.53 \times 10^{-4}$ & $6.51 \times 10^{-3}$ & $2.16 \times 10^{-4}$ & 881 \\
\hline 6 & 0.05 & -2.18 & 0.0497 & 0.0031 & 0.0051 & $1.30 \times 10^{-3}$ & $8.54 \times 10^{-3}$ & $2.28 \times 10^{-4}$ & 2002 \\
\hline 7 & 0.05 & -2.184 & 0.0496 & 0.00101 & 0.0021 & $1.96 \times 10^{-3}$ & $1.09 \times 10^{-2}$ & $2.97 \times 10^{-4}$ & 4957 \\
\hline 8 & 0.05 & -2.185 & 0.0502 & 0.00047 & 0.00105 & $2.24 \times 10^{-3}$ & $1.17 \times 10^{-2}$ & $3.30 \times 10^{-4}$ & 10101 \\
\hline 9 & 0.05 & & 0.0498 & & & $2.53 \times 10^{-3}$ & $1.25 \times 10^{-2}$ & $3.91 \times 10^{-4}$ & $\infty$ \\
\hline 10 & 0.1 & -1.60 & 0.0783 & 0.4319 & 0.333 & $8.11 \times 10^{-5}$ & $2.56 \times 10^{-3}$ & $1.57 \times 10^{-4}$ & 207 \\
\hline 11 & 0.1 & -1.80 & 0.0903 & 0.2810 & 0.391 & $1.50 \times 10^{-4}$ & $4.00 \times 10^{-3}$ & $2.39 \times 10^{-4}$ & 300 \\
\hline 12 & 0.1 & -1.90 & 0.0953 & 0.2087 & 0.294 & $2.46 \times 10^{-4}$ & $3.83 \times 10^{-3}$ & $2.34 \times 10^{-4}$ & 488 \\
\hline 13 & 0.1 & -2.00 & 0.1007 & 0.1236 & 0.242 & $3.23 \times 10^{-4}$ & $5.03 \times 10^{-3}$ & $2.28 \times 10^{-4}$ & 734 \\
\hline 14 & 0.1 & -2.10 & 0.0990 & 0.0496 & 0.132 & $6.35 \times 10^{-4}$ & $6.98 \times 10^{-3}$ & $2.21 \times 10^{-4}$ & 1235 \\
\hline 15 & 0.1 & -2.18 & 0.1000 & 0.00337 & 0.0147 & $1.55 \times 10^{-3}$ & $1.13 \times 10^{-2}$ & $3.58 \times 10^{-4}$ & 11491 \\
\hline 16 & 0.1 & & 0.0997 & & & $2.11 \times 10^{-3}$ & $1.20 \times 10^{-2}$ & $3.93 \times 10^{-4}$ & $\infty$ \\
\hline
\end{tabular}

and $S_{\infty}$. The initializing fully turbulent flow is a wellstudied fluid dynamics problem known as "open channel flow" (Pope 2000). The flow quickly becomes stratified with a fresh, cold layer forming under the ice (Fig. 1). The run is continued to an equilibrated state where the time-averaged melt rate and all other flow properties are statistically steady, which generally took $\sim 50 \mathrm{~h}$ of model time. For several runs with very strong thermal driving the flow approached equilibrated state very slowly and had not equilibrated even after $400 \mathrm{~h}$. These runs are referred to as quasi-equilibrated. Once in equilibrated state the simulations are run for a further $10 \mathrm{~h}$ to allow time-averaging of statistical properties. The quasi-equilibrated runs generally require a longer averaging interval of $>50 \mathrm{~h}$ (as discussed in section 4).

\section{Scaling theory}

\section{a. Viscous, conductive, and diffusive sublayer scaling}

Immediately below the ice is a viscous sublayer where the flow is laminar. A conductive temperature and a diffusive salinity sublayer also form below the ice. The viscous, conductive and diffusive sublayer scalings are

$$
U^{+} \sim z^{+}, \quad T^{+} \sim z^{+} \operatorname{Pr}, \quad S^{+} \sim z^{+} \mathrm{Sc},
$$

where the distance, velocity, temperature, and salinity are expressed in wall units (indicated by the plus superscript),

$z^{+}=\frac{z u_{*}}{\nu}, \quad U^{+}=\frac{U}{u_{*}}, \quad T^{+}=\frac{\left(T-T_{b}\right)}{T_{*}}, \quad S^{+}=\frac{\left(S-S_{b}\right)}{S_{*}}$

and $T_{*}=\kappa_{T}|\partial T / \partial z|_{b} / u_{*}$ and $S_{*}=\kappa_{S}|\partial S / \partial z|_{b} / u_{*}$ are the friction temperature and salinity, respectively (where the boundary values and gradients are calculated from the formulated boundary conditions in appendix $C$ ). The conductive and diffusive sublayers are thinner than the viscous sublayer because the diffusivities of heat and salt are smaller than viscosity $(\mathrm{Pr}, \mathrm{Sc}>1)$.

\section{b. Law-of-the-wall and Monin-Obukhov scaling}

Further away from the ice, at the edge of the viscous layer, small-scale turbulent structures form and drive larger-scale turbulent eddies in the "surface layer." The solid boundary of the ice influences the size of the turbulent eddies in the surface layer. When the effects of stratification are weak, the shear $\partial U / \partial z$ is expected to depend on the strength of turbulence (in the form of the friction velocity $u_{*}$ ) and the distance from the boundary $z$. Dimensional analysis then gives $\partial U / \partial z \sim u_{*} / z$, known as the law-of-the-wall scaling.

For stratified flow, Monin-Obukhov theory predicts similarity between the form of the shear and the vertical scalar gradients as

$$
\frac{\partial U}{\partial z}=\frac{u_{*}}{k_{m} z} \Phi_{m}(\xi), \quad \frac{\partial T}{\partial z}=\frac{T_{*}}{k_{s} z} \Phi_{s}(\xi), \quad \frac{\partial S}{\partial z}=\frac{S_{*}}{k_{s} z} \Phi_{s}(\xi),
$$

where $k_{m}=0.41$ and $k_{s}=0.48$ are the von Kármán constants for the momentum and scalars, respectively, following Bradshaw and Huang (1995). The MoninObukhov functions $\Phi_{m}$ and $\Phi_{s}$ are dependent on the normalized distance from the ice $\xi=z / L$, where $L$ is the Obukhov length,

$$
L=-\frac{u_{*}^{3}}{k_{m} B},
$$


and the vertical buoyancy flux at the ice-ocean interface is $B=g\left(\alpha \kappa_{T}|\partial T / \partial z|_{b}-\beta \kappa_{S}|\partial S / \partial z|_{b}\right)$. When stratification is weak, $\Phi_{m}=\Phi_{s}=1$ and Eq. (14) reverts to the law-ofthe-wall scaling.

For flow that is strongly affected by stratification, the form of the Monin-Obukhov function is still debated, with significant work done on this question in the atmospheric boundary layer community (e.g., Businger et al. 1971; Kaimal et al. 1976; Foken 2006). One common form is a linear function of $\xi$,

$$
\Phi_{m}(\xi)=1+\beta_{m} \xi, \quad \Phi_{s}(\xi)=1+\beta_{s} \xi,
$$

where the constants are $\beta_{m}=4.8$ and $\beta_{s}=5.6$ (Wyngaard 2010; Zhou et al. 2017).

Integrating Eqs. (14) with (16) and writing in terms of wall units,

$$
\begin{aligned}
U^{+} & =\frac{1}{k_{m}} \ln \left(z^{+}\right)+\frac{\beta_{m}}{k_{m}} \xi+C_{m}, \\
T^{+} & =\frac{1}{k_{s}} \ln \left(z^{+}\right)+\frac{\beta_{s}}{k_{s}} \xi+C_{T}, \quad \text { and } \\
S^{+} & =\frac{1}{k_{s}} \ln \left(z^{+}\right)+\frac{\beta_{s}}{k_{s}} \xi+C_{S},
\end{aligned}
$$

where the constant $C_{m}=5.0$, following Bradshaw and Huang (1995). The scalar $C_{T}$ and $C_{S}$ are theorized to be functions of $\mathrm{Pr}$ and Sc, respectively. The form (e.g., Schlichting and Gersten 2003)

$$
C_{T}=13.7 \mathrm{Pr}^{2 / 3}-7.5, \quad C_{S}=13.7 \mathrm{Sc}^{2 / 3}-7.5,
$$

has been found to work well for stratified plane Couette flow with Prandtl number around unity (Deusebio et al. 2015; Zhou et al. 2017). Kader and Yaglom (1972) derived a very similar expression to Eq. (20) for flow past a hydraulically smooth boundary, but with slightly different constant values (see discussions in McPhee et al. 1987; Holland and Jenkins 1999).

\section{c. Three-equation parameterization}

A common parameterization for the dynamics in the entire surface layer, including the sublayers and melt condition, is the three-equation model (McPhee et al. 1987; Holland and Jenkins 1999). The turbulent fluxes of heat and salt toward the ice are parameterized by heat $\Gamma_{T}$ and salt $\Gamma_{S}$ transfer coefficients multiplied by the friction velocity. The three equations are then the conservation of heat and salt,

$$
\begin{aligned}
c_{w} \rho_{w} u_{*} \Gamma_{T}\left(T_{\infty}-T_{b}\right) & =\rho_{i} L_{i} m, \\
\rho_{w} u_{*} \Gamma_{S}\left(S_{\infty}-S_{b}\right) & =\rho_{i} S_{b} m,
\end{aligned}
$$

respectively, and the liquidus condition [Eq. (11)]. The three-equation model was first conceptualized in terms of $u_{*}$ (McPhee et al.1987). However, for use in a system with only far-field velocity data available, a drag coefficient $C_{d}=\left(u_{*} / u_{\infty}\right)^{2}$ can be introduced to act as the third undetermined coefficient, resulting in

$$
\begin{gathered}
c_{w} \rho_{w} C_{d}^{1 / 2} u_{\infty} \Gamma_{T}\left(T_{\infty}-T_{b}\right)=\rho_{i} L_{i} m, \\
\rho_{w} C_{d}^{1 / 2} u_{\infty} \Gamma_{S}\left(S_{\infty}-S_{b}\right)=\rho_{i} S_{b} m .
\end{gathered}
$$

In the observational context, the far-field velocity $u_{\infty}$ is the free-stream current below the surface layer that is independent of the distance from the ice, with the farfield temperature and salinity measured at the same depth. Values for $\Gamma_{T}, \Gamma_{S}$, and $C_{d}$ must be prescribed in this model. Observations from beneath the Ronne ice shelf give drag coefficient $C_{d}=0.0097$, heat transfer coefficient $\Gamma_{T}=0.011$, and salt transfer coefficient $\Gamma_{S}=3.1 \times 10^{-4}$ (Jenkins et al. 2010).

The diffusive conservation equations at the boundary in Eqs. (9)-(10) coupled with the three-equation conservation equations (21)-(22) give, by definition (McPhee 2008),

$\Gamma_{T}=\frac{\kappa_{T}\left|\frac{\partial T}{\partial z}\right|_{b}}{u_{*}\left(T_{\infty}-T_{b}\right)}=\frac{1}{T_{\infty}^{+}}, \quad \Gamma_{S}=\frac{\kappa_{S}\left|\frac{\partial S}{\partial z}\right|_{b}}{u_{*}\left(S_{\infty}-S_{b}\right)}=\frac{1}{S_{\infty}^{+}}$,

where $T_{\infty}^{+}$and $S_{\infty}^{+}$are the normalized temperature and salinity differences [Eq. (13)] between the ice and the far field. Similarly the drag coefficient is

$$
C_{d}=\left(\frac{u_{*}}{U_{\infty}}\right)^{2}=\left(\frac{1}{U_{\infty}^{+}}\right)^{2}
$$

where $U_{\infty}^{+}$is the normalized far-field velocity.

\section{Results}

\section{a. Mean flow properties and melt rate}

Vertical profiles of horizontally averaged velocity, temperature, and salinity show the influence of the imposed far-field temperature on the flow structure (Fig. 2). Immediately below the ice lies the interfacial sublayer where the viscous scaling is consistent with the measured velocities (Fig. 2a). The lower edge of the viscous boundary layer is an important region for the formation of small-scale turbulent phenomena which go on to produce turbulence throughout the flow. Farther away from the ice, the case with weaker thermal driving (dark blue line) has a velocity profile similar to the logarithmic law-of-the-wall scaling (dashed) 


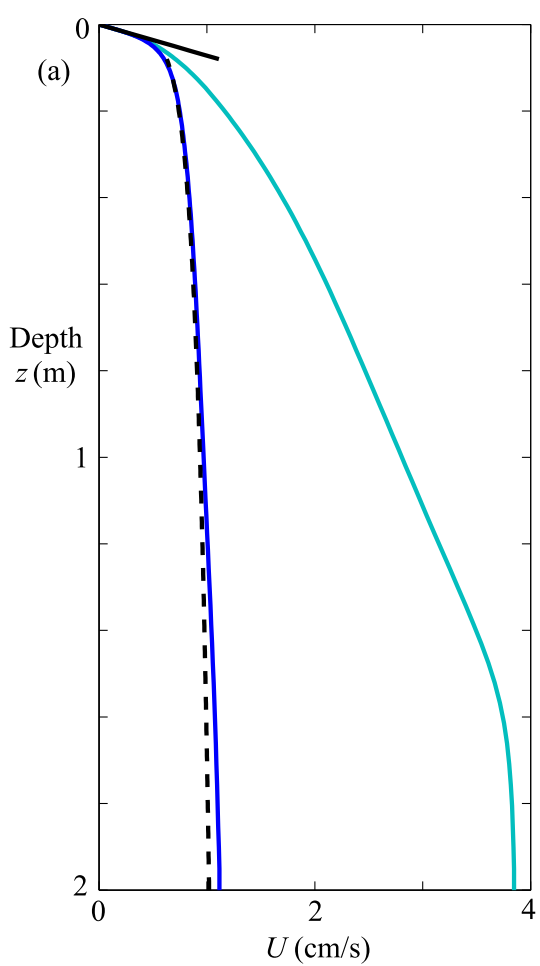

(b)
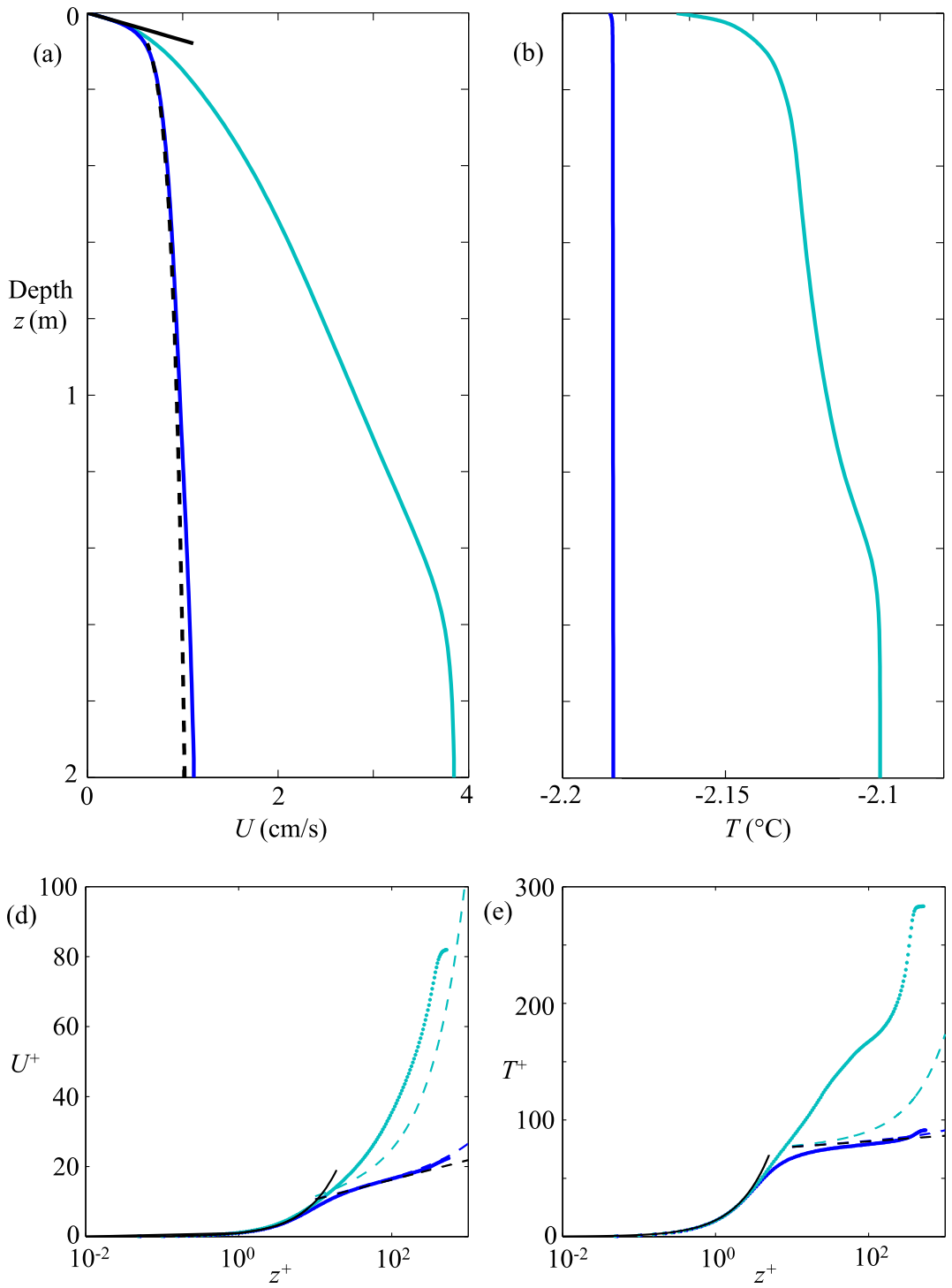

(e)

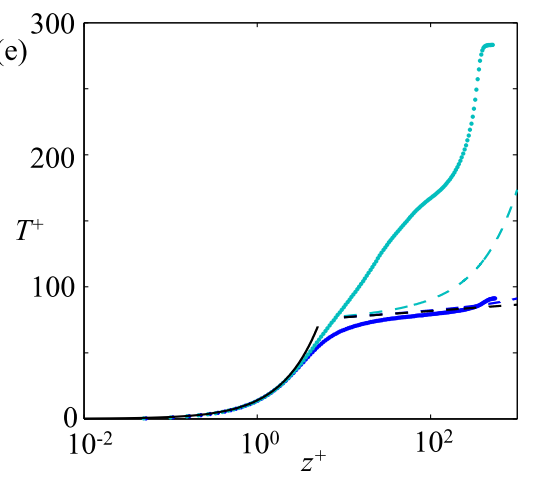

(c)

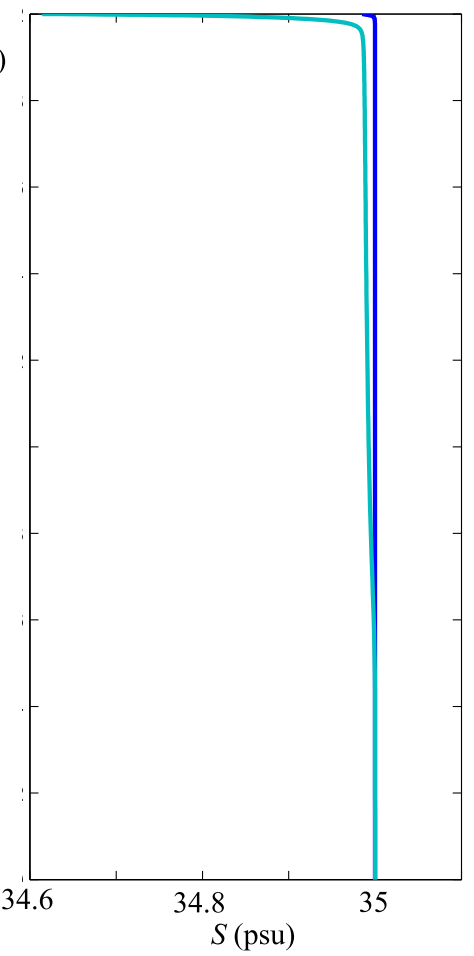

(f)

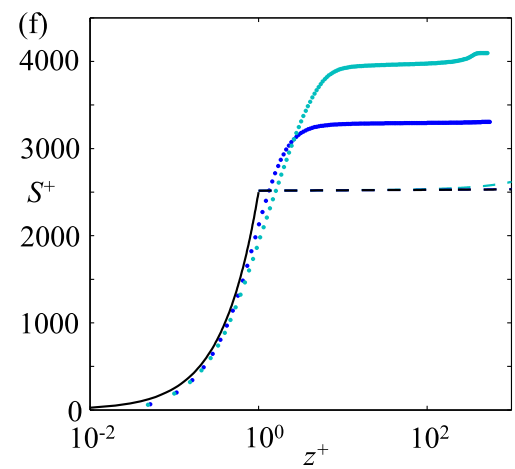

FIG. 2. Vertical profiles of $u_{*}=0.05 \mathrm{~cm} \mathrm{~s}^{-1}$ cases with weak thermal driving $\Delta T=0.00101^{\circ} \mathrm{C}$ (run 7 ; blue) and strong thermal driving $\Delta T=0.0641^{\circ} \mathrm{C}$ (run 3 ; cyan). The results are taken in equilibrated (or quasi-equilibrated) state and are horizontally averaged across the domain and time-averaged for $>50 \mathrm{~h}$ (run 3) and $10 \mathrm{~h}$ (run 7). The profiles are (a) velocity, (b) temperature, and (c) salinity with depth. Wall-normalized profiles of (d) velocity $U^{+}$, (e) temperature $T^{+}$, and (f) salinity $S^{+}$are shown against depth in wall units $z^{+}$. In (d)-(f) the spacing of the symbols indicates the grid spacing. The viscous, conductive, and diffusive boundary layer scalings [Eq. (12)] are shown as the unbroken black lines. The Monin-Obukhov scalings [Eqs. (17)-(19)] are shown as the broken lines colored to match the runs, and the black broken curve indicates the passive scalar case.

but with a modest increase in the far-field velocity. The increase in far-field velocity is very large in the case with stronger thermal forcing (cyan line). Increases in far-field temperature lead to a stronger temperature stratification (Fig. 2b) and hence larger thermal driving. This increases the melt rate, freshening the water and producing a stronger salinity stratification (Fig. 2c). The density stratification is dominated by the salinity component in all the runs presented here. Hence, the stabilizing salinity stratification damps out some of the small-scale turbulence at the edge of the viscous boundary layer, and as a result the drag decreases. However, as the friction velocity is prescribed (via imposing the pressure gradient) the equilibrated state wall shear stress must remain the same no matter the imposed far-field temperature, and so the reduction in drag results in an acceleration of the far-field velocity.

Vertical profiles of velocity, temperature, and salinity are plotted in terms of wall units in Figs. $2 \mathrm{~d}-\mathrm{f}$ where the results all closely match their respective sublayer 

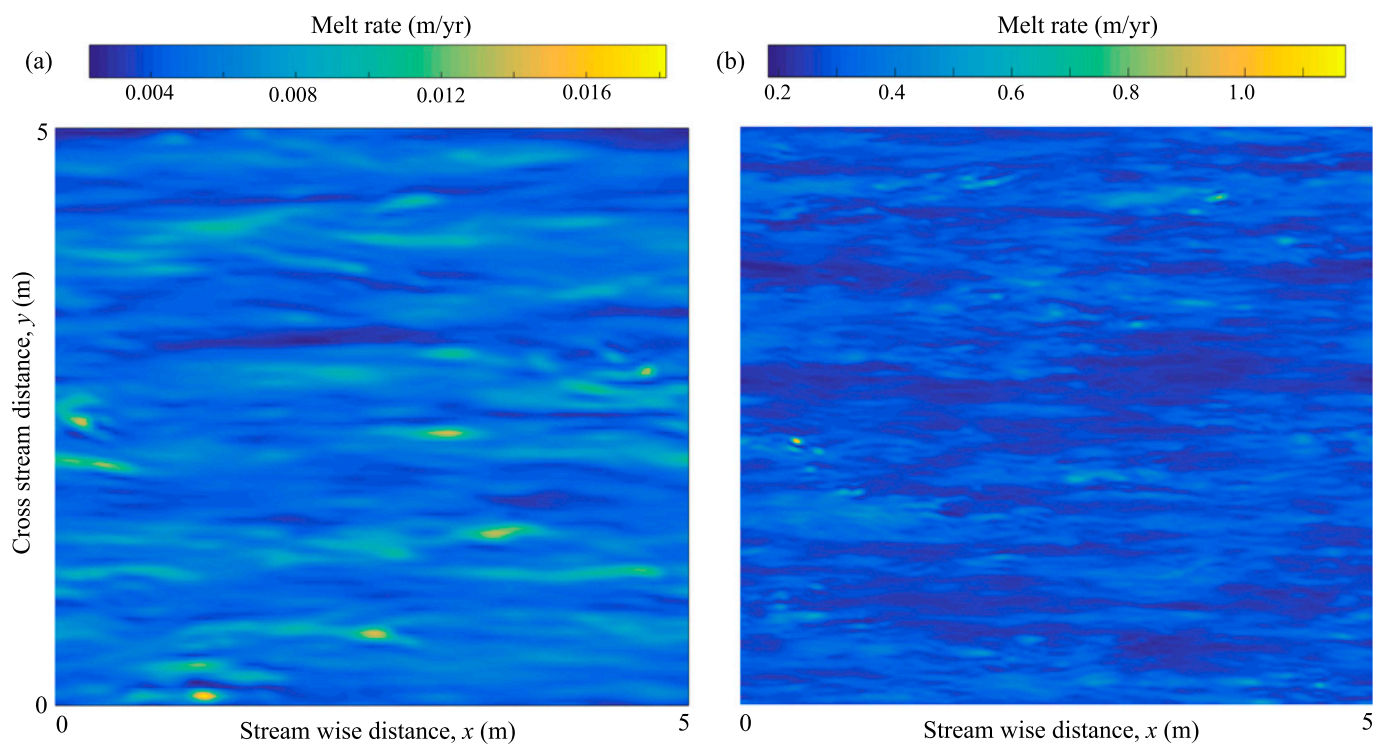

FIG. 3. Snapshots of the melt rate at the base of the ice for two weak thermal driving cases with (a) $u_{*}=0.05 \mathrm{~cm} \mathrm{~s}^{-1}$, $\Delta T=0.0031^{\circ} \mathrm{C}$ (run 6) and (b) $u_{*}=0.1 \mathrm{~cm} \mathrm{~s}^{-1}, \Delta T=0.1236^{\circ} \mathrm{C}($ run 13$)$.

scalings, indicating that the resolution is sufficient to fully resolve these sublayers. It is important to adequately resolve the sublayers to ensure that the resulting melt rate is correct. The Monin-Obukhov scaling in Eqs. (17)-(19) does reasonably well predicting the velocity profiles, even when the flow is strongly influenced by the stratification (Fig. 2d). The scaling is consistent with the temperature profile for weak stratification but departs significantly from the strongly stratified profile (Fig. 2e). For the salinity profiles, the scaling is reasonable for the passive scalar results (not shown here) but departs from the LES results for even the most weakly stratified case (Fig. 2f). Note that the Monin-Obukhov scaling for the salinity profile in Eq. (19) is dominated by the huge Schmidt number in the $C_{S}$ term and is barely influenced by the stratification term $\left(\beta_{s} \xi / k_{s}\right)$. A further Schmidt number dependence could be introduced in the MoninObukhov scaling for strong stratification, to adjust the scaling when the stratifying element has molecular diffusivity much smaller than the molecular viscosity. However, it is beyond the scope of this paper to derive a new scaling.

At the ice base there can be large instantaneous spatial variability in the melt rate (Fig. 3) with peaks of up to 5 times the mean. These peaks are correlated with smallscale turbulent structures that form at the edge of the viscous boundary layer. Turbulent structures such as near-wall streaks are effective at transporting heat across the viscous boundary layer and hence a signature of these structures appears in the melt rate snapshots.

The mean melt rate is shown in Fig. 4 for all the runs in Table 1 . The melt rates have been horizontally averaged across the ice base and averaged in time for $10 \mathrm{~h}$, except for runs 1-3 and 10-11 which were averaged for $>50 \mathrm{~h}$. The passive scalar cases $(g=0)$ are included as lines in Fig. 4 since these results apply for any imposed $\Delta T$ (for a particular $\left.u_{*}\right)$. This is because the advection-diffusion equation [Eq. (3)] is linear in temperature and, for the passive scalar case, there is no influence of the stratification on the flow, meaning that the melt rate in Eq. (9) is also a linear function of the temperature gradient. This is consistent with the Monin-Obukhov scaling in Eq. (18),

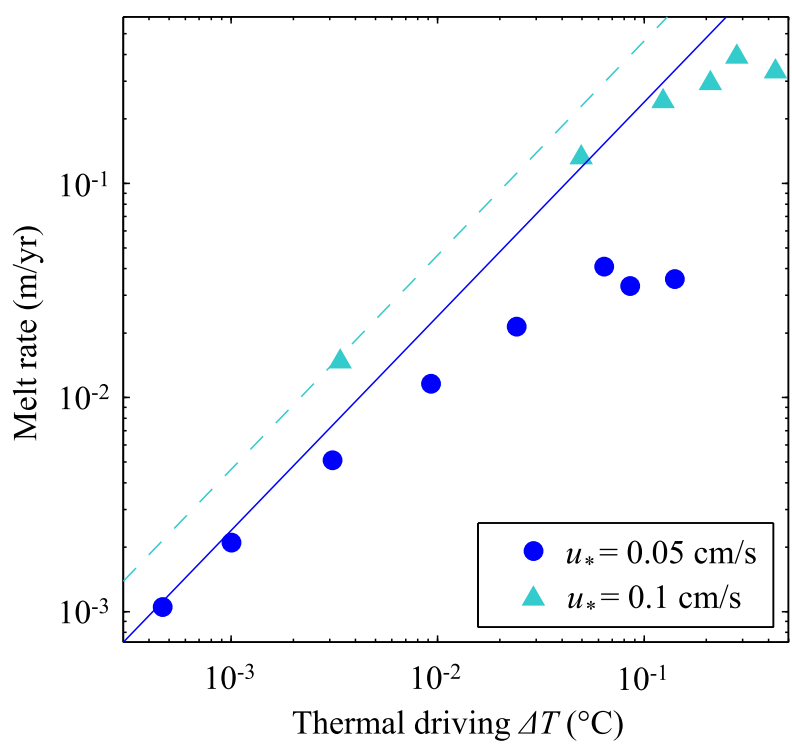

FIG. 4. Melt rate against thermal driving for all runs in Table 1. The passive scalar $g=0$ cases with $u_{*}=0.05 \mathrm{~cm} \mathrm{~s}^{-1}$ (run 9; unbroken line) and $u_{*}=0.1 \mathrm{~cm} \mathrm{~s}^{-1}$ (run 16; broken line) are also shown. 


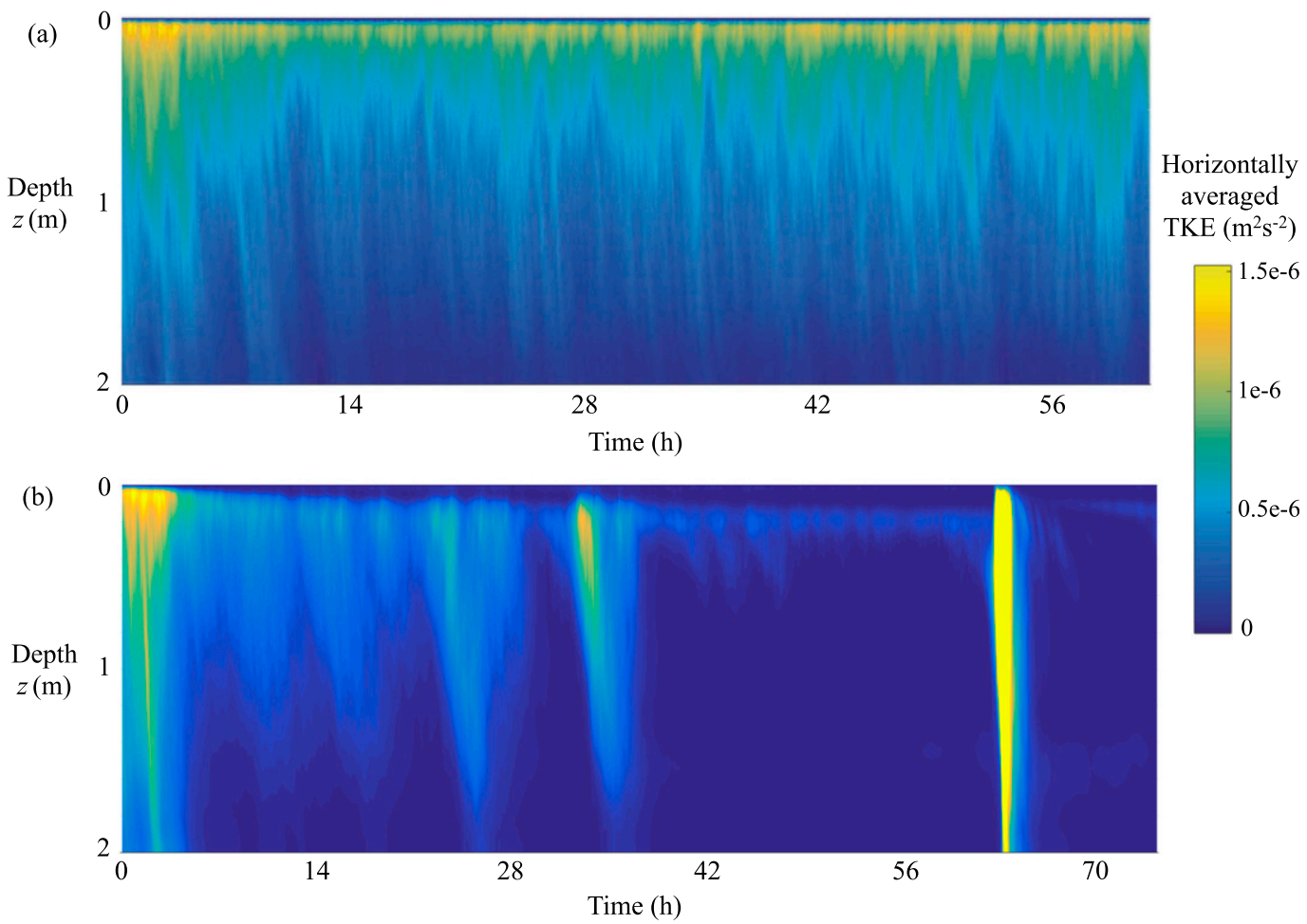

FIG. 5. Adjustment of the turbulent kinetic energy $\left(\mathrm{m}^{2} \mathrm{~s}^{-2}\right)$ from fully developed unstratified turbulence to turning on the melt condition. The evolution is shown for $u_{*}=0.05 \mathrm{~cm} \mathrm{~s}^{-1}$ with (a) weak thermal driving $\Delta T=$ $0.0031^{\circ} \mathrm{C}$ (run 6) and (b) strong thermal driving $\Delta T=0.0641^{\circ} \mathrm{C}$ (run 3). Note the different time windows shown.

which predicts that when the scalar is passive (stratification term $\left.\beta_{s} \xi / k_{s}=0\right)$ and $u_{*}$ unchanged, the wall-normalized temperature at a particular depth $T^{+}$is constant. Therefore increases in imposed $\Delta T$ are compensated for by a linear increase in $\partial T /\left.\partial z\right|_{b}$ and hence a linear increase in the melt rate [Eq. (9)]. The passive scalar simulations were used to calculate the $\Gamma_{T}=1 / T_{\infty}^{+}$associated with each $u_{*}$ case (runs 9 and 16 in Table 1) which, using Eq. (21), resulted in the lines on Fig. 4.

For stronger thermal driving, the melt rate departs from the value for passive scalars as the stable stratification inhibits turbulence and its ability to mix heat toward the boundary and melt the ice. At very strong thermal driving, the melt rates appear to become largely independent of $\Delta T$. The point at which thermal driving and the stable salinity stratification become strong enough to damp turbulence is dependent on the friction velocity-higher friction velocities have more energetic turbulence and so stronger stratification is required to reduce the heat transfer and melt rate.

\section{b. Evolution of boundary layer turbulence}

The response of the flow at early times in the simulations (Fig. 5) provides insight into the boundary layer turbulence. Recall that the initial condition consists of fully turbulent flow with uniform temperature and salinity, $T_{\infty}$ and $S_{\infty}$. After a few hours, the flow becomes stratified in temperature and salinity and the stable stratification acts to reduce the turbulent kinetic energy (TKE) at the edge of the viscous layer. For weak thermal driving the flow remains turbulent and reaches the equilibrated state after $\sim 50 \mathrm{~h}$ (Fig. 5a). When thermal driving is strong, the stratification damps the turbulence for long periods of time between episodic turbulent events (Fig. 5b). These intermittent cases do not reach equilibrated state in $50 \mathrm{~h}$ and must be continued for long periods of time to equilibrate.

One intermittently turbulent case (run 3 ) is shown in more detail in Fig. 6 to better understand the nature of the turbulent bursts. The TKE and friction velocity are both small during intervals of laminar flow before rapidly increasing when the flow goes turbulent. The bulk flow accelerates when the flow is laminar and the turbulence and friction velocity are small and exert less drag on the far-field current. The trace of TKE through time with friction velocity and driving temperature (Fig. 6g) begins when the flow is laminar. At the time immediately before a turbulent burst $(t=338.3 \mathrm{~h})$ the stratification near the ice is very weak (Fig. 6f), allowing turbulent structures to form at the edge of the viscous 

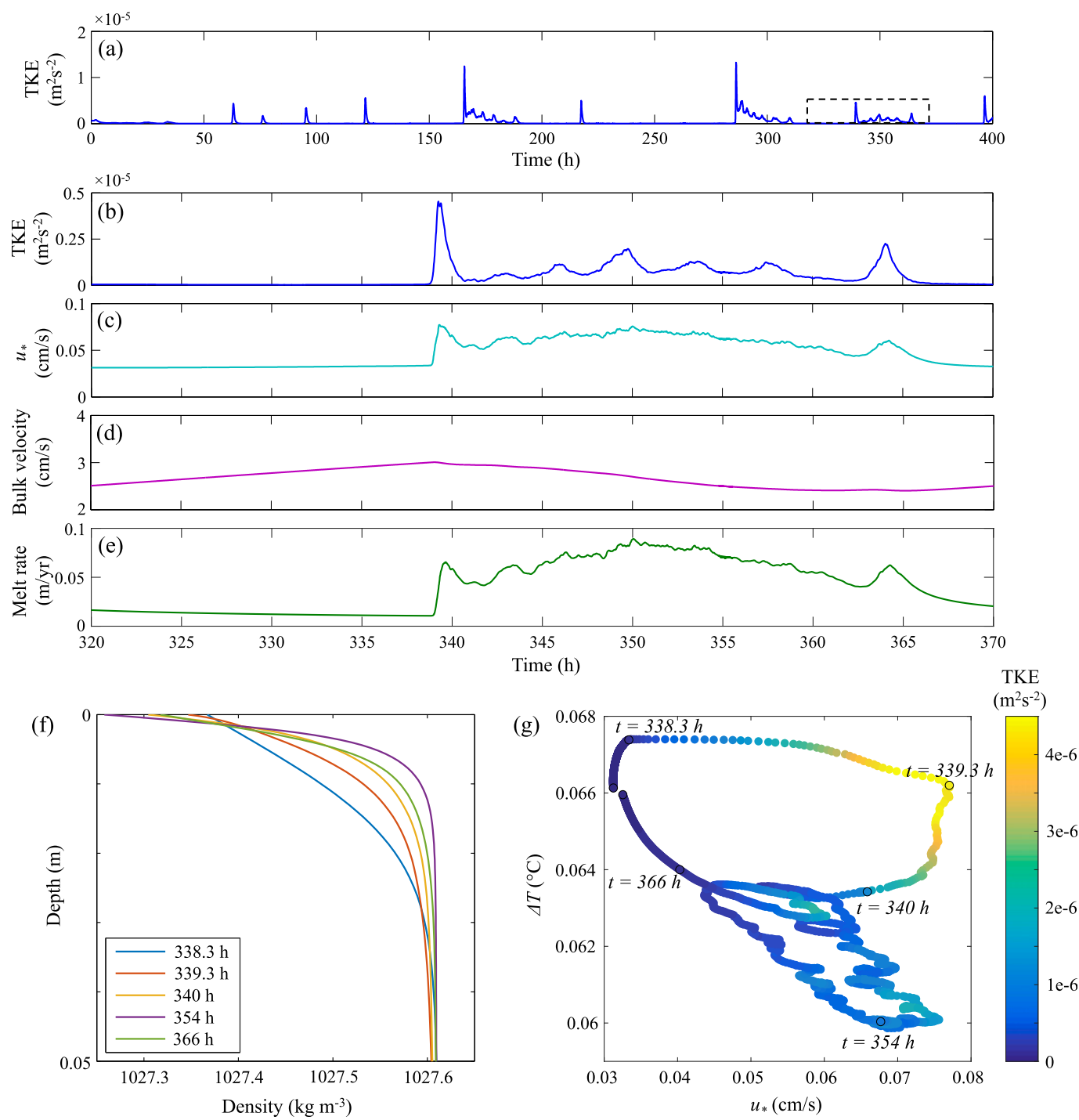

FIG. 6. Laminar to turbulent transition for imposed $u_{*}=0.05 \mathrm{~cm} \mathrm{~s}^{-1}$ with strong thermal driving (time-averaged $\Delta T=0.0641^{\circ} \mathrm{C}$; run 3). (a) Volume-averaged turbulent kinetic energy with time, where the dotted box shows zoom in on an interval of (b) volume-averaged turbulent kinetic energy, (c) friction velocity $u_{*}$, (d) bulk velocity, and (e) melt rate. (f) Density at the top region of the domain, immediately beneath the ice-ocean boundary at various times, and $(\mathrm{g})$ the progression of the thermal driving and friction velocity through time, with the color bar showing the volume-averaged turbulent kinetic energy.

sublayer. When a turbulent burst begins, the friction velocity and TKE rapidly increase to their maximum values $(t=339.3 \mathrm{~h})$. The turbulence mixes more heat across the sublayer, increasing the temperature at the ice base $T_{b}$ while decreasing $\Delta T=T_{\infty}-T_{b}$ (at $\left.t=340 \mathrm{~h}\right)$. The melt rate increases in response to the increase in heat. In the salinity field (which dominates the density) the increased melt rate results in a decrease in the salinity at the boundary, resulting in a decrease in density near the ice as shown in Fig. 6f. As the turbulence continues, the density at the boundary reduces further until eventually the stable stratification is strong enough to damp turbulence. The trailing edge of the loop at smaller $\Delta T(t=354 \mathrm{~h})$ shows the continued smaller levels of turbulence which eventually die out as the system becomes laminar again. As the turbulence intensity decreases, less heat is transferred to the ice and so $\Delta T$ begins to slowly increase $(t=366 \mathrm{~h})$. The density at the boundary slowly increases toward the preturbulent maximum, weakening the stratification under the ice again to eventually set off another turbulent burst.

Similar turbulent events occur in runs 1,2,10, and 11, although when thermal driving is very strong the turbulent portion of the trajectory in $\Delta T, u_{*}$ space is shorter as 
turbulence dies out more quickly. In terms of time scales, these bursts occur quasi-regularly every $50 \mathrm{~h}$ or so, with similar time scales in runs 1 and 2 . While similar turbulent bursts occur for simulations with imposed $u_{*}=0.1 \mathrm{~cm} \mathrm{~s}^{-1}$ that have large thermal driving (runs 10 and 11) it is more computationally expensive to run these for long intervals, hence there are fewer events to examine and the time interval of reoccurrence is unclear. The intermittently turbulent runs show that the TKE is not just a function of friction velocity and that the time history matters.

In an effort to quantify whether the system is fully or intermittently turbulent, we calculate the time-averaged TKE along with the standard deviation away from this mean (Fig. 7). For the smaller thermal driving (runs 4-8 and 12-15), the flow is fully turbulent and the TKE has small standard deviation. For larger thermal driving, the standard deviation increases significantly and there is a decrease in the total TKE as the flow becomes intermittently turbulent.

\section{c. Three-equation parameterization and Obukhov length}

Here we examine whether the turbulent fluxes can be approximated by transfer coefficients as assumed in the three-equation model. For each simulation the drag coefficient in Eq. (26) and the transfer coefficients for heat $\Gamma_{T}$ and salt $\Gamma_{S}$ in Eq. (25) are calculated. As thermal driving increases, all coefficients decrease as the flow becomes less turbulent (Fig. 8). The exception is the salt transfer coefficient which has a short plateau when moving from fully turbulent to intermittent flow. The ratio of $\Gamma_{T} / \Gamma_{S}=34$ in Fig. $8 \mathrm{~d}$ matches the more turbulent simulations and is broadly consistent with past predictions of $\Gamma_{T} / \Gamma_{S}$ between 35 and 70 (McPhee et al. 2008).

The passive scalar cases are shown as horizontal lines in Figs. 8a-c. There is very little dependence of $\Gamma_{T}$ and $\Gamma_{S}$ on the friction velocity for the passive scalar cases. The drag coefficient decreases by a small amount with increasing friction velocity, which is a known result for turbulent channel flow (Dean 1978; Pope 2000). The lack of dependence of $\Gamma_{T}$ and $\Gamma_{S}$ on the friction velocity suggests that constant transfer coefficients are a good approximation for strongly turbulent flow. It also begs the question of whether there is a normalizing factor that would collapse the results when the flow is less strongly turbulent and allow prediction of whether the flow will be turbulent or intermittent.

The Obukhov length in Eq. (15) can be interpreted as the distance away from the ice where stratification begins to strongly affect the flow. For a distance much larger than the Obukhov length $(z \gg L)$ stratification strongly affects the flow. Conversely for $z \ll L$ stratification effects are weak. Molecular viscosity is important in the viscous sublayer that extends to approximately $50 \delta_{\nu}$, where $\delta_{\nu}=\nu / u_{*}$ is the viscous length scale (Pope

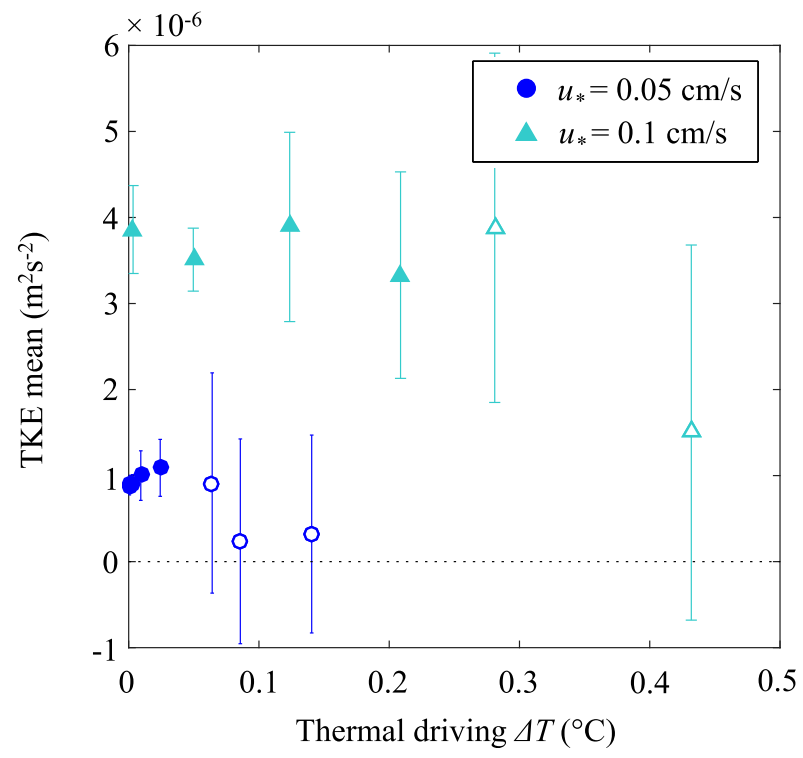

FIG. 7. Turbulent kinetic energy against thermal driving. Results have been time averaged for $10 \mathrm{~h}$, excepting cases where the turbulence was intermittent (the higher $\Delta T$ values shown by open symbols) where the flow was averaged for longer $(>50 \mathrm{~h})$ to achieve accurate representation of the flow becoming turbulent and then relaminarizing, as shown in Fig. 6. The vertical bars show the standard deviation of the turbulent kinetic energy around the mean, and the dotted line shows the zero turbulent kinetic energy value. Closed symbols show runs that are fully turbulent, and open symbols show runs that are intermittently turbulent.

2000). We can define the frictional Obukhov length as the ratio of $L$ to the viscous length scale,

$$
L^{+}=L / \delta_{\nu}
$$

When $L^{+}$is sufficiently small there is no region of the flow where turbulence is free from the suppressing effects of stratification or viscosity. Previous work has found that flow in a stratified boundary layer becomes laminar when $L^{+}<100$ (Flores and Riley 2011). Simulations of stratified plane Couette flow indicate that the flow is fully turbulent when $L^{+}>200$ and intermittently turbulent when $100<L^{+}<200$ (Deusebio et al. 2015). It is worth noting that there is some ambiguity on how to define the thickness of the viscous boundary layer, as the effects of viscosity continue to decrease moving away from the boundary (Pope 2000). This leads to some ambiguity in the $L^{+}$thresholds, so they should be interpreted as general guidelines rather than definitive regime changes.

The variance in TKE is plotted as a function of the time-averaged $L^{+}$(from calculating $L^{+}$at each time step and then time averaging) in Fig. 9. For large $L^{+}$, stratification effects are weak and the flow is fully turbulent with small variance around the mean TKE. As $L^{+}$decreases, the variance in TKE increases as the flow 
(a)

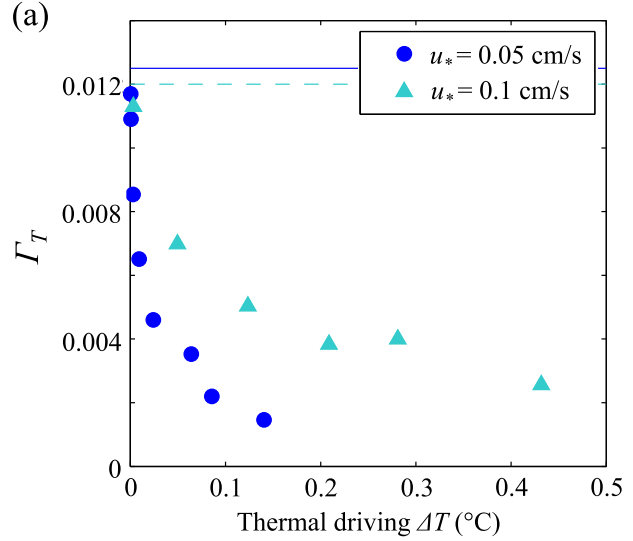

(c)

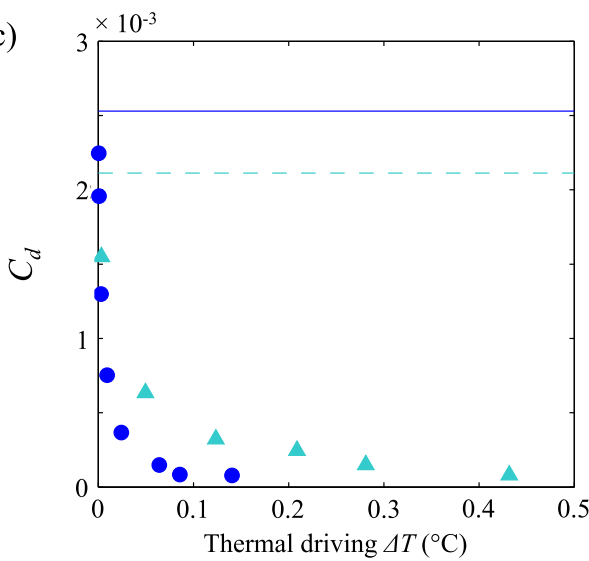

(b)

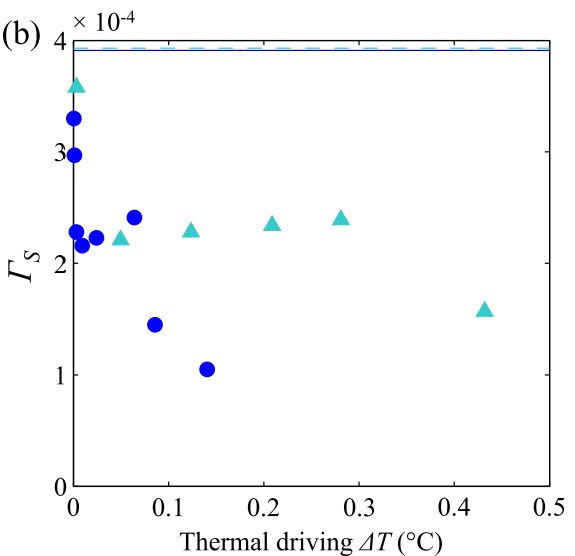

(d)

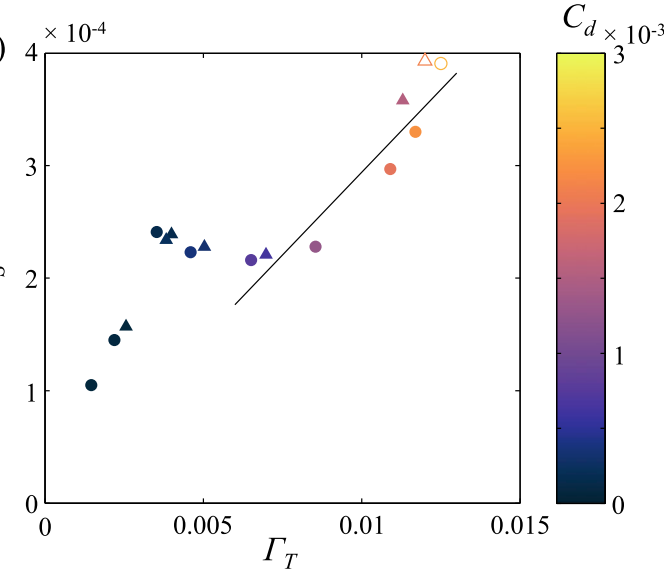

FIG. 8. Transfer coefficients of (a) heat $\Gamma_{T}$ and (b) salt $\Gamma_{S}$, and (c) drag coefficient $C_{d}$ against thermal driving. The lines on (a)-(c) show the passive scalar $g=0$ cases (run 9 , unbroken line and run 16 , broken line). (d) The variation of $\Gamma_{S}$ with $\Gamma_{T}$, with $C_{d}$ shown on the color bar. Open symbols are the passive scalar cases, and the curve is fitted to the fully turbulent cases with a slope of $1 / 34$.

becomes intermittently turbulent and eventually laminar for long intervals with turbulent bursts. Note that here the time-averaged $L^{+}$is larger than 200 even for large thermal driving (see Table 1) because of the feedback effect between the melt condition and the stable stratification (as discussed in detail in section $4 \mathrm{~b}$ ). Stratified flows without this feedback can reach less than 100 and become completely laminar (Deusebio et al. 2015; Zhou et al. 2017). The frictional Obukhov length $L^{+}$generally does well describing the transition from turbulent to intermittent flow in the ISOBL, although there appears to be some remaining dependence of the TKE variance on $u_{*}$.

Crucially, $L^{+}$collapses the transfer coefficients for different imposed friction velocities (Fig. 10). The drag coefficients for different friction velocities do not fully collapse, partly because the passive scalar values vary with friction velocity. Normalizing by the passive scalar values improves the collapse of the $u_{*}$ curves as a function of $L^{+}$(Fig. 10d). Also included in Fig. 10 is the Monin-Obukhov similarity scaling prediction for the coefficients. Far-field values of $U^{+}, T^{+}$, and $S^{+}$(defined in 13) are solved for using the Monin-Obukhov similarity scaling [Eqs. (17-19)] as functions of $L^{+}$and $u_{*}$. The resulting $U_{\infty}^{+}, T_{\infty}^{+}$, and $S_{\infty}^{+}$are used to calculate the transfer [Eq. (25)] and drag [Eq. (26)] coefficients. The Monin-Obukhov prediction is reasonably consistent with the diagnosed $C_{d}$ and $\Gamma_{T}$ (Fig. 10). However, the Monin-Obukhov similarity scaling does not capture the dependence of $\Gamma_{S}$ on $L^{+}$. Note that other suggestions for constant values in the Monin-Obukhov scaling were also tested (e.g., Kader and Yaglom 1972; McPhee et al. 1987) but the presented scaling with constants from Schlichting and Gersten (2003) showed the best fit to the simulations.

For fully turbulent flow with large $L^{+}$, the transfer coefficients asymptote to the upper limit given by the passive scalar case $\left(\Gamma_{T}=0.012\right.$ and $\left.\Gamma_{S}=3.9 \times 10^{-4}\right)$. 


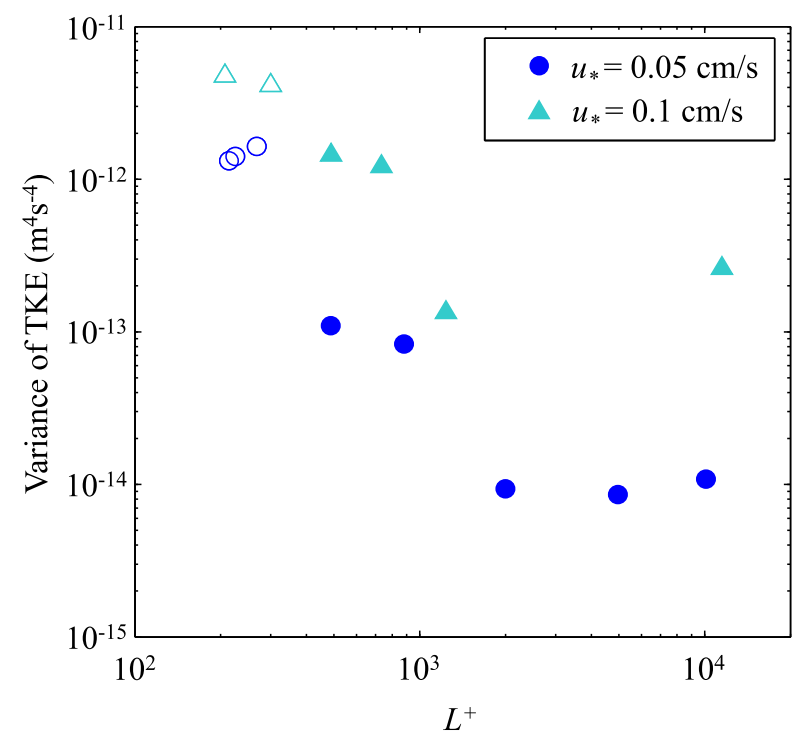

FIG. 9. Variance in turbulent kinetic energy against the ratio of Obukhov to viscous length scale $L^{+}$. The time interval considered was $10 \mathrm{~h}$, except in cases when the turbulence was intermittent where the flow was averaged for longer $(>50 \mathrm{~h})$ as in Fig. 7. Closed symbols show runs that are fully turbulent, and open symbols show runs that are intermittently turbulent.

These results are very similar to observations $\left(\Gamma_{T}=0.011\right.$ and $\Gamma_{S}=3.1 \times 10^{-4}$ ) by Jenkins et al. (2010). That the $\Gamma_{T}$ and $\Gamma_{S}$ results for different $u_{*}$ collapse to the same $L^{+}$ curves is evidence that these results may apply to a larger range of $u_{*}$ and $\Delta T$. Using the maximum limiting values of $\Gamma_{T}=0.012$ and $\Gamma_{S}=3.9 \times 10^{-4}$, the three-equation model in Eqs. (21)-(22) can be solved to predict the melt rate and $S_{b}$ as functions of both $\Delta T$ and $u_{*}$. These melt rate and $S_{b}$ values are then used in the molecular flux equations (9)-(11) to give the buoyancy flux, yielding a prediction of $L^{+}$as a function of $\Delta T$ and $u_{*}$. The predicted $L^{+}$from the three-equation model (colored background) is compared against the time-averaged $L^{+}$ from the LES (symbols) in Fig. 11a. Similarly, Fig. 11b compares the predicted melt rate from the three-equation model (colored background) with the time-averaged melt rate from the LES (symbols). The size of the symbols is proportional to the TKE variance with larger symbols corresponding to high levels of TKE variance (from Fig. 9) and intermittent turbulence, while small symbols indicate low TKE variance and fully turbulent flow. In the fully turbulent simulations, the measured $u_{*}$ matches the expected $u_{*}$ (set by imposing the pressure gradient) because the flow has come to equilibrated state. For intermittently turbulent flow, the evolution to equilibrated state was extremely long $(>400 \mathrm{~h})$ hence the simulations were cut off and considered quasi-equilibrated-these cases have measured $u_{*}$ that do not yet match the imposed $u_{*}$.
The $L^{+}=100$ and $L^{+}=200$ contours are highlighted on Fig. 11 to show the predicted regime transitions. They curve upward at very strong thermal driving $\left(\Delta T \approx 5^{\circ} \mathrm{C}\right)$ where the heat flux starts to noticeably contribute to the buoyancy flux in $L^{+}$. Following the $L^{+}=200$ contour, the maximum predicted melt rate for a turbulent flow is then $0.05 \mathrm{~m} \mathrm{yr}^{-1}$ for $u_{*}=0.05 \mathrm{~cm} \mathrm{~s}^{-1}$ and $0.9 \mathrm{~m} \mathrm{yr}^{-1}$ for $u_{*}=0.1 \mathrm{~cm} \mathrm{~s}^{-1}$, the latter of which is close to geophysically relevant values (Nicholls et al. 2009; Kimura et al. 2015). Comparing the predicted $L^{+}=200$ contour with the simulation results shows that our approach does well predicting the transition from fully turbulent to intermittently turbulent flow. The simulated flow does not become fully laminar for predicted $L^{+}<100$ but remains intermittently turbulent even at very strong thermal driving. The measured $L^{+}$in Table 1 are calculated using a long time-average that, when the flow is intermittently turbulent, includes laminar and turbulent events. Hence, the mean $L^{+}$remains above about 200, even when thermal driving is large and the $L^{+}$from the three-equation model is predicted to be less than 100 . Instantaneously, smaller values of $\mathrm{L}^{+}$occur in the LES.

Using smaller values of either $\Gamma_{T}$ or $\Gamma_{S}$ in the threeequation model results in a shift of the predicted $L^{+}$ transition curves to the left on Fig. 11 (not shown here). Physically this is because a decrease in $\Gamma_{T}$ means less heat transferred to melt the ice, while a decrease in $\Gamma_{S}$ means less salt and hence a higher melting temperature, both of which result in smaller melt rates and a decrease in the stabilizing stratification that suppresses turbulence. As mentioned previously, there is some ambiguity in the onset of intermittent flow, which is not necessarily abrupt. The $L^{+}=100,200$ predictions are not hard transitions but more general guidelines on when the flow might be expected to be fully turbulent. As such, using the upper limits on $\Gamma_{T}$ and $\Gamma_{S}$ indicates the area where the threeequation model works (with these specific upper limit values of the transfer coefficients) and when it has the potential to not work well. The prediction matches well with the change from fully to intermittently turbulent flow found in the simulations, where the level of turbulence in the simulations is indicated by the size of the symbols in Fig. 11 (with smaller symbols corresponding to more turbulent flow).

The three-equation model with the upper limits of $\Gamma_{T}$ and $\Gamma_{S}$ also does well predicting the melt rate for the fully turbulent cases (Fig. 12). But, as we might expect when using the large values of the transfer coefficients, it overestimates the melt rate by almost an order of magnitude for the intermittently turbulent simulations. One extension to this work could be to incorporate the dependence of $\Gamma_{T}, \Gamma_{S}$ on $L^{+}$(seen in Fig. 10) into the threeequation model to improve the predicted melt rate when 
(a)

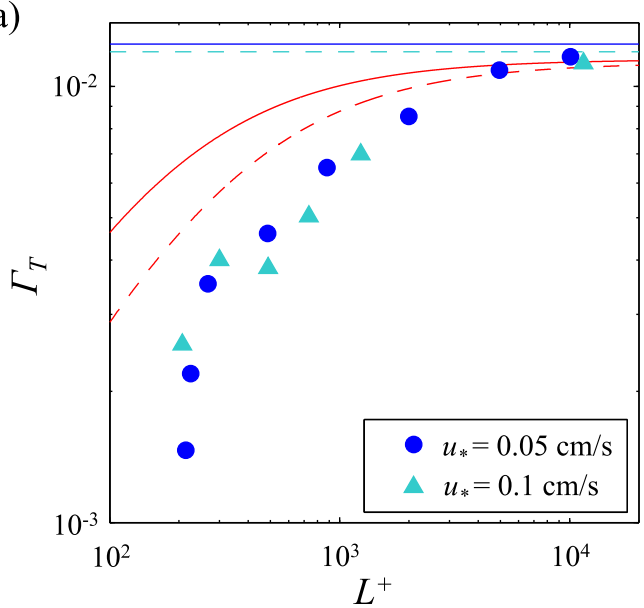

(c)

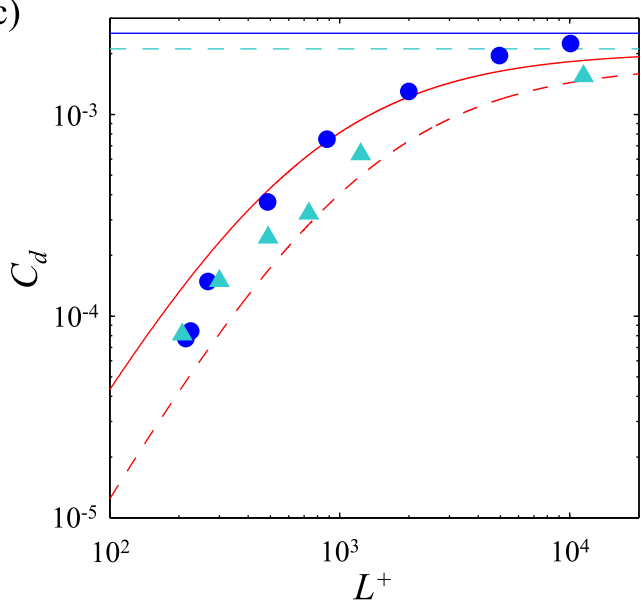

(b)

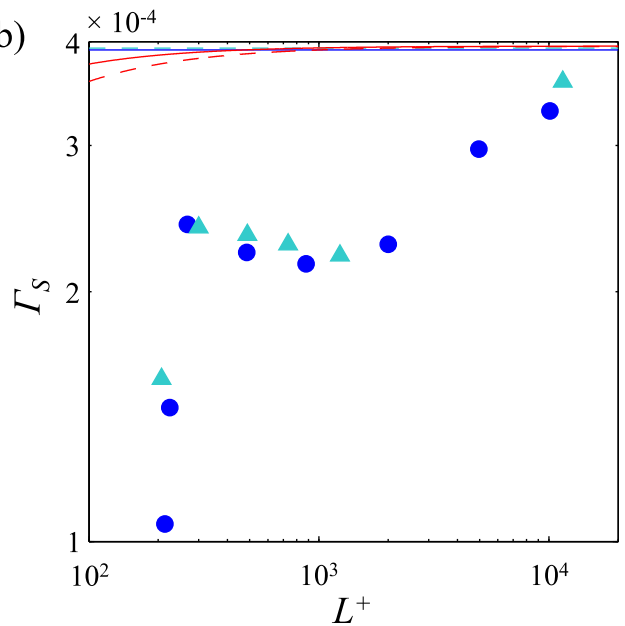

(d)

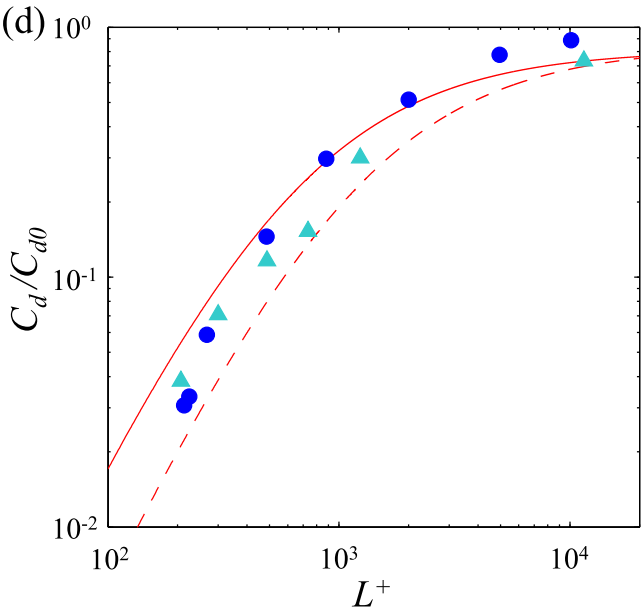

FIG. 10. Transfer coefficients of (a) heat $\Gamma_{T}$ and (b) salt $\Gamma_{S}$, and (c) drag coefficient $C_{d}$ against Obukhov length scale ratio $L^{+}$. (d) The drag coefficient has been normalized by that measured for the passive scalar case. The lines are for the passive scalar $g=0$ cases (run $9 u_{*}=0.05 \mathrm{~cm} \mathrm{~s}^{-1}$, blue unbroken and run $16 u_{*}=0.1 \mathrm{~cm} \mathrm{~s}^{-1}$, cyan broken) and for the Monin-Obukhov similarity scaling [Eqs. (17)-(19)] coupled with Eqs. (25) and (26) to predict the transfer coefficients $\left(u_{*}=0.05 \mathrm{~cm} \mathrm{~s}^{-1}\right.$, red unbroken and $u_{*}=0.1 \mathrm{~cm} \mathrm{~s}^{-1}$, red broken).

flow is intermittently turbulent. The Monin-Obukhov scaling (red lines on Fig. 10) is already reasonably successful at predicting the drop-off in $\Gamma_{T}$ and $C_{d}$ but with some deficiency in the prediction of $\Gamma_{S}$. Improving the Monin-Obukhov scaling or finding another parameterization that captures $\Gamma_{T}, \Gamma_{S}$, and $C_{d}$ behavior would be useful, but is beyond the scope of the current paper. As it stands, caution should be used when trying to apply constant transfer coefficients to a flow that is not fully turbulent.

\section{Summary}

Large-eddy simulations were used to model the upper region of the ocean boundary layer beneath a melting ice shelf. Increases in thermal driving enhance the melt rate until the flow becomes strongly stratified in salinity. Turbulence is then suppressed by the stable stratification and no longer efficiently mixes heat across the interfacial sublayer, causing the melt rate to plateau with further increases in thermal driving. At this point the flow becomes intermittently turbulent in time, with long periods of laminar flow followed by abrupt turbulent bursts.

The transition between turbulent and intermittent regimes is well-described by the ratio of the Obukhov and viscous layer thicknesses $L^{+}$. Monin-Obukhov similarity scaling for stratified flow does reasonably well predicting the drag and heat transfer coefficients for the three-equation parameterization as the simulations move into intermittent turbulence. For the salt transfer coefficient, the Monin-Obukhov scaling is consistent with 

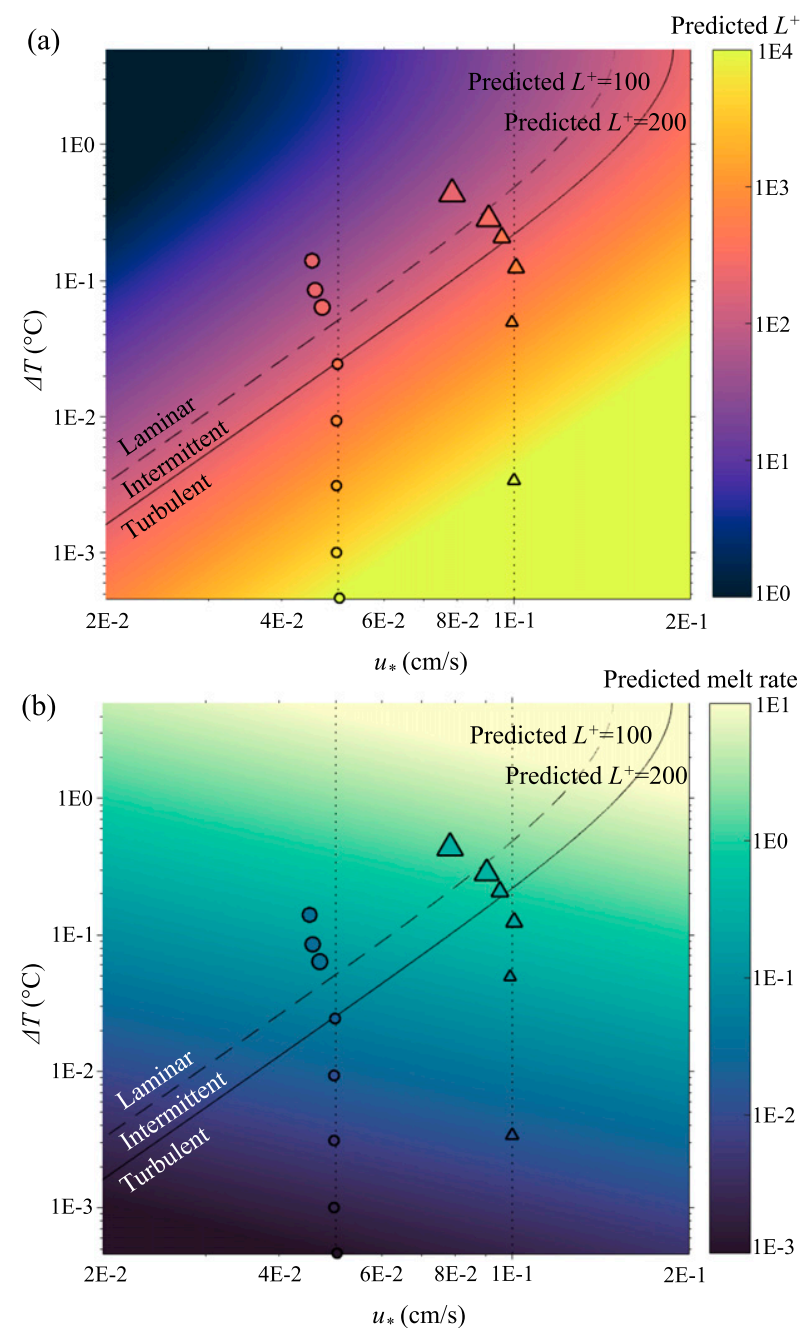

FIG. 11. Predicted (a) Obukhov to viscous length scale ratio $L^{+}$ and (b) melt rate $\left(\mathrm{m} \mathrm{yr}^{-1}\right)$ varying with friction velocity $u_{*}$ and thermal driving $\Delta T$. Color contours show (a) $L^{+}$values and (b) melt rates predicted by the three-equation model with $\Gamma_{T}=$ 0.012 and $\Gamma_{S}=3.9 \times 10^{-4}$ (the maximum limiting values found in the simulations). The black lines highlight the $L^{+}=100$ (dashed) and $L^{+}=200$ (unbroken) contours. The $u_{*}=0.05 \mathrm{~cm} \mathrm{~s}^{-1}$ (circles) and $u_{*}=0.1 \mathrm{~cm} \mathrm{~s}^{-1}$ (triangles) results are calculated from the LES, with measured values of $u_{*}$ on the horizontal axis. The dotted lines show the equilibrated state values of $u_{*}=0.05 \mathrm{~cm} \mathrm{~s}^{-1}$ and $u_{*}=$ $0.1 \mathrm{~cm} \mathrm{~s}^{-1}$. The LES that have measured $u_{*}$ less than the dotted line have not yet come to equilibrated state. The size of the symbol reflects the amount of variance in TKE, with lower variance (smaller symbols) found for more turbulent runs as in Fig. 9.

the weakly stratified simulations, but overestimates the coefficient when the stratification is strong and the turbulence becomes intermittent. Crucially, the transfer coefficients asymptote at large $L^{+}$(fully turbulent flow) for simulations with different friction velocities, giving us confidence to extend the simulated results to larger friction velocities and thermal driving that may be more geophysically relevant. These upper limits on the

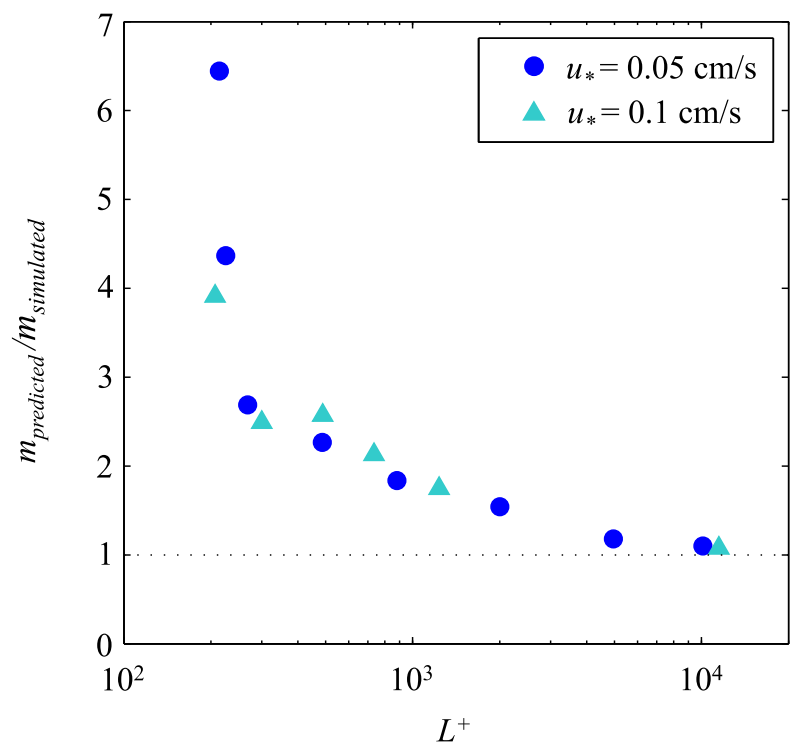

FIG. 12. The ratio of the melt rate predicted by the threeequation model to that measured in the simulations, against $L^{+}$. As in Fig. 11, the maximum limiting transfer coefficients found in the simulations $\Gamma_{T}=0.012$ and $\Gamma_{S}=3.9 \times 10^{-4}$ are used in the threeequation model.

transfer coefficients are also consistent with observed ice shelf values.

The $L^{+}$transition can be used to predict when the three-equation model (with upper limit values of transfer coefficients) is likely to work well in observations and ocean models. Understanding the direct influence of stratification induced by melting on shear driven turbulence, and the consequent feedback on the melt rate, is essential to improving parameterizations in ocean models and planning for future climate scenarios.

\section{Discussion}

Applying the $L^{+}$regime prediction to the upper region of the deeper planetary boundary layer in realworld scenarios will help to anticipate when the threeequation parameterization will work in observations and ocean models. The thermal driving and friction velocities inferred from observations are generally larger than those explored here using large-eddy simulations. Simulations with larger friction velocity are computationally expensive due to increasing grid resolution requirements. Nevertheless, because the simulated results collapse for different $u_{*}$ and approach limiting values of transfer coefficients at large $L^{+}$, the flow regime prediction has been extended to a wider range of parameters in Fig. 13 to allow comparison with observed conditions. For $u_{*}>0.2 \mathrm{~cm} \mathrm{~s}^{-1}$ the flow remains turbulent even at large thermal driving. 


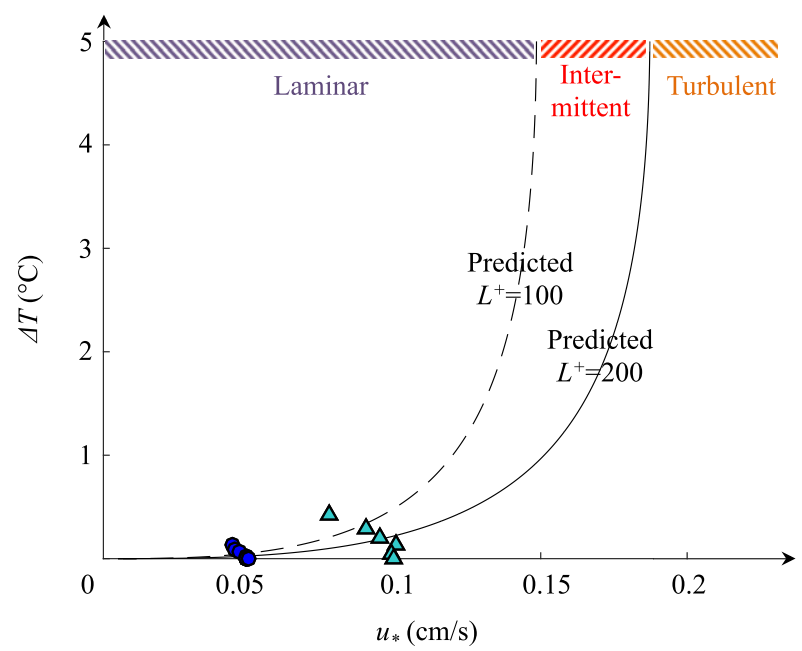

FIG. 13. Regime diagram showing the predicted transition between laminar, intermittent, and fully turbulent flow with friction velocity $u_{*}$ and thermal driving $\Delta T$. The curves show the $L^{+}=100$ (broken) and $L^{+}=200$ (unbroken) contours predicted by the threeequation model with $\Gamma_{T}=0.012$ and $\Gamma_{S}=3.9 \times 10^{-4}$ (the maximum limiting values found in the simulations). The $u_{*}=0.05 \mathrm{~cm} \mathrm{~s}^{-1}$ (circles) and $u_{*}=0.1 \mathrm{~cm} \mathrm{~s}^{-1}$ (triangles) results are calculated from the LES, with measured values of $u_{*}$ on the horizontal axis.

The Obukhov to viscous length ratio $L^{+}=L / \delta_{v}$ is connected to the mixing length scale $\lambda$ that has been used in ice-ocean studies (McPhee 2008). The mixing length is hypothesized to increase with depth until it saturates at a maximum value $\lambda_{\max }$. Stratification causes the flow in the boundary layer to become laminar when $\lambda_{\max }<R_{c} k_{m} L$, where $R_{c} \approx 0.2$ is the critical flux Richardson number (McPhee 2008). Following the arguments in section $4 \mathrm{c}$, for turbulence to exist (in the wall-bounded shear flow examined here) the mixing length must be much larger than the viscous length $\lambda_{\max } \gg \delta_{\nu}$. The mixing length condition then requires $L^{+} \gg 1 /\left(R_{c} k_{m}\right)$ or $L^{+}=12.5$ for turbulence. Requiring at least an order of magnitude difference between the mixing and viscous length scales results in $L^{+}=125$ being the minimum value of $L^{+}$for which the flow can be turbulent. This regime transition is consistent with the $L^{+}=100$ transition predicted by comparing the Obukhov layer thickness to the thickness of the viscous layer (Flores and Riley 2011). Again, we note that past work on stratified boundary layers has found completely laminar flow for $L^{+}<100$, but here the feedback between turbulence, stratification, and ice melting keeps the simulated flow intermittently turbulent.

The turbulent transfer coefficients for heat and salt diagnosed from our fully turbulent simulations with weak stratification are in good agreement with those empirically inferred from beneath the Ronne ice shelf (Jenkins et al. 2010). The drag coefficient is a factor of three smaller in the simulations compared to the Ronne ice shelf observations. This could be due to additional processes such as ice roughness which can increase the friction velocity or because, as Jenkins et al. (2010) notes, the drag coefficient is less well constrained than the transfer coefficients for this set of observations. Observations of turbulent flow under sea ice also give transfer coefficients consistent with the simulations (Sirevaag 2009). Note that the friction velocity (or drag coefficient) needs to be prescribed in the three-equation model, but it is difficult to observe and can vary significantly in space and time. Uncertainty around the friction velocity is perhaps the most difficult step in applying our results to observations or ice-melt parameterizations in ocean models.

The turbulent transfer and drag coefficients in the LES are consistent with those predicted by MoninObukhov similarity scaling, but the scaling significantly overestimates the salt transfer in stratified conditions. An improved model may require a modification to the Monin-Obukhov function $\Phi_{s}$ [see Eq. (16)] to address this additional stratification effect when the Prandtl/ Schmidt number is large. Additionally, a roughness length scale can be included in the Monin-Obukhov similarity scaling in place of the viscous length scale (e.g., Yaglom and Kader 1974).

The intermittently turbulent simulations are thought to be dynamically different from the highly stratified ISOBL observed in the ocean. This is because the prescribed pressure gradient in the simulations accelerates the far-field current for cases with strong thermal driving. In contrast, the strongly stratified flow under the George VI ice shelf is observed to have low current speeds with evidence for double-diffusive steps (Kimura et al. 2015). Work in the atmospheric boundary layer community may give insight into other dynamical processes that could become important when the flow is strongly stably stratified (see review by Mahrt 2014).

Our focus has been on simulating regions of ice shelves that do not have a significant slope. In the weakly sloped case of a few degrees away from the horizontal, plume theory predicts that there will be negligible effects of an upslope current (Kerr and McConnochie 2015; McConnochie and Kerr 2018). Here, small slopes were found to have very little effect on the flow turbulence (see appendix A), making our results applicable to small slope angles. Steeper slopes occur near the grounding line which is an important region for ice-sheet dynamics. In such cases an upslope plume may be the primary source of turbulence and is likely to influence ice-ocean interactions (McConnochie and Kerr 2017; Mondal et al. 2019). 
TABLE A1. Summary of additional runs with slope of ice changed from horizontal. Parameters are as in Table 1 with magnitude and direction of slope change also indicated.

\begin{tabular}{|c|c|c|c|c|c|c|c|c|c|c|}
\hline Run & $\begin{array}{c}\text { Slope } \\
\theta\end{array}$ & $\begin{array}{c}u_{*} \text { set } \\
\left(\mathrm{cm} \mathrm{s}^{-1}\right)\end{array}$ & $\begin{array}{c}T_{\infty} \\
\left({ }^{\circ} \mathrm{C}\right)\end{array}$ & $\begin{array}{c}u_{*} \text { measured } \\
\left(\mathrm{cm} \mathrm{s}^{-1}\right)\end{array}$ & $\Delta T\left({ }^{\circ} \mathrm{C}\right)$ & $\begin{array}{l}\text { Melt rate } \\
\left(\mathrm{m} \mathrm{yr}^{-1}\right)\end{array}$ & $C_{d}$ & $\Gamma_{T}$ & $\Gamma_{S}$ & $L^{+}$ \\
\hline 4 & None & 0.05 & -2.15 & 0.0499 & 0.0242 & 0.0214 & $3.68 \times 10^{-4}$ & $4.60 \times 10^{-3}$ & $2.23 \times 10^{-4}$ & 486 \\
\hline $4 \mathrm{~A}$ & $1^{\circ}$ in $x$ & 0.05 & -2.15 & 0.0498 & 0.0248 & 0.0221 & $3.62 \times 10^{-4}$ & $4.70 \times 10^{-3}$ & $2.43 \times 10^{-4}$ & 469 \\
\hline $4 B$ & $1^{\circ}$ in $y$ & 0.05 & -2.15 & 0.0501 & 0.0246 & 0.0222 & $3.57 \times 10^{-4}$ & $4.70 \times 10^{-3}$ & $2.39 \times 10^{-4}$ & 476 \\
\hline $4 \mathrm{C}$ & $5^{\circ}$ in $x$ & 0.05 & -2.15 & 0.0500 & 0.0253 & 0.0219 & $3.44 \times 10^{-4}$ & $4.50 \times 10^{-3}$ & $2.50 \times 10^{-4}$ & 482 \\
\hline 4D & $5^{\circ}$ in $y$ & 0.05 & -2.15 & 0.0507 & 0.0248 & 0.0229 & $3.74 \times 10^{-4}$ & $4.70 \times 10^{-3}$ & $2.46 \times 10^{-4}$ & 486 \\
\hline 8 & None & 0.05 & -2.185 & 0.0502 & 0.00047 & 0.00105 & $2.24 \times 10^{-3}$ & $1.17 \times 10^{-2}$ & $3.30 \times 10^{-4}$ & 10101 \\
\hline $8 \mathrm{~A}$ & $1^{\circ}$ in $x$ & 0.05 & -2.185 & 0.0501 & 0.00044 & 0.00106 & $2.23 \times 10^{-3}$ & $1.18 \times 10^{-2}$ & $3.37 \times 10^{-4}$ & 9861 \\
\hline $8 \mathrm{~B}$ & $1^{\circ}$ in $y$ & 0.05 & -2.185 & 0.0499 & 0.00044 & 0.00106 & $2.22 \times 10^{-3}$ & $1.18 \times 10^{-2}$ & $3.38 \times 10^{-4}$ & 9760 \\
\hline 13 & None & 0.1 & -2.00 & 0.1007 & 0.1236 & 0.242 & $3.23 \times 10^{-4}$ & $5.03 \times 10^{-3}$ & $2.28 \times 10^{-4}$ & 734 \\
\hline $13 \mathrm{~A}$ & $1^{\circ}$ in $x$ & 0.1 & -2.00 & 0.1004 & 0.1244 & 0.239 & $3.11 \times 10^{-4}$ & $5.00 \times 10^{-3}$ & $2.29 \times 10^{-4}$ & 733 \\
\hline $13 \mathrm{~B}$ & $1^{\circ}$ in $y$ & 0.1 & -2.00 & 0.1008 & 0.1240 & 0.240 & $3.16 \times 10^{-4}$ & $5.00 \times 10^{-3}$ & $2.27 \times 10^{-4}$ & 742 \\
\hline
\end{tabular}

The present study was motivated by the ice shelf/ocean boundary layer. However, many results from the simulations can apply more generally to other ice-ocean interactions including land-fast and drifting sea ice. The formation of ice from seawater can result in a small ice salinity, commonly observed to be 3-7 psu for land-fast ice (Gerland et al. 1999; Vancoppenolle et al. 2007). Increasing the ice salinity in the simulations from the fresh ice shelf to saltier fast ice values is expected to modestly increase the melting temperature, but otherwise the results and conclusions will be very similar. It would be reasonably straightforward to include a constant $S_{\text {ice }}$ value in the melting equation [Eq. (10)]. Drifting ice can generate shear-driven turbulence as it moves across the ocean, but this could be modeled in a reference frame moving with the ice with a possibly time-dependent current imposed in the ocean. Perhaps the most problematic assumption made here when applied to sea ice is the assumption that the ice-ocean interface is flat and smooth. It is possible to include a roughness length in the Monin-Obukhov scaling (Yaglom and Kader 1974), but large roughness elements such as leads and ice keels would be more challenging to simulate.

Future work will focus on simulations with larger thermal driving and friction velocities to get closer to realworld scenarios. There are also many other processes that are likely to affect the melt rate such as roughness of the ice, tides and basal slope. The simulations here were designed to model a subset of the larger planetary boundary layer-future work could include Earth's rotation and to have both a surface layer and an outer layer. While it is significantly more difficult to simulate, the changing topography of the melting ice and the formation of channel cavities will be important in directing the melt outflow. We have not considered effects such as allowing the thermal expansion coefficient to vary with temperature, however this is unlikely to have much influence unless temperature differences become large. Other complicated flow phenomena such as double-diffusive layers will also be relevant for ice melting.

Acknowledgments. The NERC Standard Grant NE/N009746/1 is gratefully acknowledged for supporting the research presented here. This work used the ARCHER U.K. National Supercomputing Service (http://www.archer.ac.uk). Supporting data such as the DIABLO source code and input files to reproduce the simulations are available at https://doi.org/10.17863/ CAM.39569. The authors are grateful to P. Holland for his helpful comments on an earlier version of the manuscript and to A. Jenkins, K. Nicholls, P. Davis, L. Couston, R. Patmore, and L. Middleton for their thoughts and discussion on this work. Thanks also to the editor and two anonymous reviewers for their comments that helped to improve the manuscript.

\section{APPENDIX A}

\section{Sloped Runs}

In additional runs, the influence of small basal slope angles on the turbulent flow is examined (Table A1). The momentum equation [Eq. (1)] was changed for a slope in the $x$ direction,

$$
\frac{D \mathbf{u}}{D t}=-\frac{1}{\rho_{0}} \nabla p+\nu \nabla^{2} \mathbf{u}+F \mathbf{i}+\frac{\Delta \rho}{\rho_{0}} g(\sin \theta \mathbf{i}+\cos \theta \mathbf{k})-\nabla \cdot \boldsymbol{\tau}
$$

or a slope in the $y$ direction,

$$
\frac{D \mathbf{u}}{D t}=-\frac{1}{\rho_{0}} \nabla p+\nu \nabla^{2} \mathbf{u}+F \mathbf{i}+\frac{\Delta \rho}{\rho_{0}} g(\sin \theta \mathbf{j}+\cos \theta \mathbf{k})-\nabla \cdot \boldsymbol{\tau} .
$$

The gravity term in Eqs. (A1) and (A2) leads to a mean component that can drive an upslope plume by forcing 
the mean momentum equation. However, we want to ensure that the only contribution to the friction velocity is from the imposed pressure gradient so that the equilibrium state friction velocity is consistent across results with different slopes. To do this, the mean density gradient in the horizontal and vertical directions is subtracted off the momentum equations [Eqs. (A1) and (A2)]. This has no effect on the stability but does not allow for the formation of an upslope plume. However, the imposed forcing $F$ can be viewed as the upslope component of an imposed hydrostatic pressure gradient. Therefore, it is just the feedback between changes in mean density and the upslope buoyancy force that are neglected. This is not expected to have a strong effect in cases with small slopes, especially where the flow is dominated by shear turbulence such as the cases examined here.

Three fully turbulent runs from Table 1 were selected as base cases, with the direction of gravity angled to produce an ice slope of either $1^{\circ}$ or $5^{\circ}$ from the horizontal in the streamwise $x$ or cross-stream $y$ direction. The tilt of gravity does not have much, if any, influence on the turbulence in this system, as is shown by the results in Table A1. Future work will be to simulate the full boundary layer including the upslope acceleration for more strongly sloped cases. We note that there can be important feedbacks between melting and slope that act on larger scales (Jenkins 2016) that has not been ruled out here.

\section{APPENDIX B}

\section{Anisotropic Minimum Dissipation Model for Large-Eddy Simulations}

The large-eddy simulations have subfilter stress tensor $\tau_{i j}=\overline{u_{i} u_{j}}-\overline{u_{i}} \overline{u_{j}}$ with the deviatoric part of the stress tensor $\tau_{i j}^{d}$ modeled as

$$
\tau_{i j}^{d}=\tau_{i j}-\frac{1}{3} e_{i j} \tau_{k k}=-2 \nu_{\mathrm{SGS}} \overline{S_{i j}},
$$

where Einstein summation is implied, $e_{i j}$ is the delta function, $\nu_{\mathrm{SGS}}$ is the subgrid scale eddy viscosity, and $\overline{S_{i j}}=(1 / 2)\left[\partial_{i} \bar{u}_{j}(x, t)+\partial_{j} \bar{u}_{i}(x, t)\right]$ is the resolved rate-ofstrain tensor. The overbar denotes filtering at the resolved spatial scale which for our purposes corresponds to the resolved grid scale. The subfilter scalar fluxes of heat $\lambda_{T, j}=\overline{u_{i} T}-\overline{u_{i}} \bar{T}$ and salt $\lambda_{S, j}=\overline{u_{i} S}-\overline{u_{i}} \bar{S}$ are modeled respectively as

$$
\lambda_{T, j}=-\kappa_{T, \mathrm{SGS}} \partial_{j} \bar{T}, \quad \lambda_{S, j}=-\kappa_{S, \mathrm{SGS}} \partial_{j} \bar{S},
$$

where $\kappa_{T, \mathrm{SGS}}$ and $\kappa_{S, \mathrm{SGS}}$ are the subgrid-scale scalar diffusivities for heat and salt respectively. For ease of reading we now drop the overbar, recalling that spatial filtering is implied.

The anisotropic minimum-dissipation (AMD) model was derived by Rozema et al. (2015). Extending this model to a stratified scenario following Abkar and Moin (2017) but modified to fulfil the Verstappen (2016) requirement (by normalizing the displacement, velocity, and the velocity gradient by the filter width $\delta$ to ensure that the resulting eddy dissipation properly counteracts the spurious kinetic energy transferred by convective nonlinearity) gives subgrid scale viscosity,

$$
\nu_{\mathrm{SGS}}=(C \delta)^{2} \frac{\max \left\{-\left(\hat{\partial}_{k} \hat{u}_{i}\right)\left(\hat{\partial}_{k} \hat{u}_{j}\right) \hat{S}_{i j}+\hat{e}_{i 3} g\left(\hat{\partial}_{k} \hat{u}_{i}\right) \hat{\partial}_{k} \rho, 0\right\}}{\left(\hat{\partial}_{l} \hat{u}_{m}\right)\left(\hat{\partial}_{l} \hat{u}_{m}\right)},
$$

where $C$ is a modified Poincaré constant,

$$
\begin{aligned}
& \hat{x}_{i}=\frac{x_{i}}{\delta_{i}}, \quad \hat{u}_{i}(\hat{x}, t)=\frac{u_{i}(x, t)}{\delta_{i}}, \quad \hat{\partial}_{i} \hat{u}_{j}(\hat{x}, t)=\frac{\delta_{i}}{\delta_{j}} \partial_{i} u_{j}(x, t), \\
& \hat{e}_{i 3}=\frac{e_{i 3}}{\delta_{3}},
\end{aligned}
$$

where $\delta_{i}$ is the filter width in the direction of $x_{i}$, and the normalized rate-of-strain tensor is

$$
\hat{S}_{i j}=\frac{1}{2}\left[\hat{\partial}_{i} \hat{u}_{j}(\hat{x}, t)+\hat{\partial}_{j} \hat{u}_{i}(\hat{x}, t)\right] .
$$

For flows that are not very strongly stratified (Vreugdenhil and Taylor 2018) the second term in Eq. (B3) is small and the subgrid-scale viscosity becomes

$$
\nu_{\mathrm{SGS}}=(C \delta)^{2} \frac{\max \left\{-\left(\hat{\partial}_{k} \hat{u}_{i}\right)\left(\hat{\partial}_{k} \hat{u}_{j}\right) \hat{S}_{i j}, 0\right\}}{\left(\hat{\partial}_{l} \hat{u}_{m}\right)\left(\hat{\partial}_{l} \hat{u}_{m}\right)} .
$$

The AMD model was extended by Abkar et al. (2016) to provide a subgrid scalar diffusivities for heat and salt

$$
\begin{aligned}
\kappa_{T, \mathrm{SGS}} & =(C \delta)^{2} \frac{\max \left\{-\left(\hat{\partial}_{k} \hat{u}_{i}\right)\left(\hat{\partial}_{k} T\right) \hat{\partial}_{i} T, 0\right\}}{\left(\hat{\partial}_{l} T\right)\left(\hat{\partial}_{l} T\right)}, \\
\kappa_{S, \mathrm{SGS}} & =(C \delta)^{2} \frac{\max \left\{-\left(\hat{\partial}_{k} \hat{u}_{i}\right)\left(\hat{\partial}_{k} S\right) \hat{\partial}_{i} S, 0\right\}}{\left(\hat{\partial}_{l} S\right)\left(\hat{\partial}_{l} S\right)} .
\end{aligned}
$$

For the filter width $\delta$ we follow the suggestion of Verstappen (2016) to use

$$
\frac{1}{\delta^{2}}=\frac{1}{3}\left(\frac{1}{\delta_{x}^{2}}+\frac{1}{\delta_{y}^{2}}+\frac{1}{\delta_{z}^{2}}\right)
$$


where the filter widths in each direction are $\left(\delta_{x}, \delta_{y}, \delta_{z}\right)$ and the Poincare constant is $C^{2}=1 / 12$. In the vertical direction, where the second-order finite differences scheme is used for the grid discretization, the filter width is defined as $\delta_{z}=\left(z_{k+1}-z_{k-1}\right)$, where $k$ is the grid cell (Verstappen 2016). In the two horizontal directions the grid is discretized using Fourier modes and a $2 / 3$ dealiasing rule is applied moving from Fourier back to physical space. The filter widths are then $\delta_{x}=(3 / 2)\left(x_{i+1}-x_{i-1}\right)=3 \Delta x$ and $\delta_{y}=(3 / 2)\left(z_{k+1}-z_{k-1}\right)=3 \Delta y$ where $i$ and $j$ are the grid cells and $\Delta x$ and $\Delta y$ are the grid cell size in each respective direction (Vreugdenhil and Taylor 2018).

\section{APPENDIX C}

\section{Implementation of Melting Boundary Conditions}

In the vertical direction $z$ the numerical solver has a grid for the vertical velocities (named $G$ for base grid) and a staggered grid (named GF for fractional grid) for the horizontal velocities and scalars (Taylor 2008). The staggered grid is halfway between neighboring points of the base grid such that, for grid point $k$, the staggered grid is $\mathrm{GF}_{k}=(1 / 2)\left(G_{k+1}+G_{k}\right)$. This staggering ensures that neighboring pressure values are coupled. The working volume is composed of $N$ grid points in the vertical direction, along with ghost cells at the base and top ( 0 and $N+1)$. We define the top of the working volume as the grid point $G_{N}$ where the vertical velocity is zero (impermeable boundary condition). As $G_{N}$ is the location of the ice base $T_{b}, S_{b}$ and the melt rate $m$ are also defined at $G_{N}$.

Recalling that the numerical discretization is secondorder finite difference in the vertical direction, the scalars and scalar gradients at the top boundary can be expressed as

$$
\begin{array}{ll}
T_{b}=T_{b, \text { int }}=\frac{1}{2}\left(T_{N}+T_{N-1}\right), & S_{b}=S_{b, \text { int }}=\frac{1}{2}\left(S_{N}+S_{N-1}\right), \\
\frac{\partial T}{\partial z}=\left(\frac{\partial T}{\partial z}\right)_{\mathrm{int}}=\frac{T_{N}-T_{N-1}}{\Delta z_{N}}, & \frac{\partial S}{\partial z}=\left(\frac{\partial S}{\partial z}\right)_{\mathrm{int}}=\frac{S_{N}-S_{N-1}}{\Delta z_{N}},
\end{array}
$$

where the subscript "int" refers to the interpolated value and $\Delta z$ is the grid spacing. The above form assumes that the vertical grid spacing of neighboring points is unity; a more accurate version could be used for highly stretched grids. The melting equations [Eqs. (9)-(11)] become

$$
\begin{aligned}
c_{w} \rho_{w} \kappa_{T}\left(\frac{\partial T}{\partial z}\right)_{\mathrm{int}} & =\rho_{i} L_{i} m, \\
\rho_{w} \kappa_{S}\left(\frac{\partial S}{\partial z}\right)_{\mathrm{int}} & =\rho_{i} S_{b, \mathrm{int}} m, \text { and }
\end{aligned}
$$

$$
T_{b, \text { int }}=\lambda_{1} S_{b, \text { int }}+\lambda_{2}+\lambda_{3} P .
$$

Each time step, $T_{N-1}(x, y)$ and $S_{N-1}(x, y)$ from the working volume are used to solve the quadratic equation resulting from Eqs. (C2)-(C4) for $T_{N}(x, y), S_{N}(x, y)$, and the melt rate $m(x, y)$. Dirichlet boundary conditions are used to implement $T_{N}(x, y)$ and $S_{N}(x, y)$ on the staggered grid, resulting in $T_{b}(x, y)$ and $S_{b}(x, y)$ at the ice boundary on the base grid.

\section{REFERENCES}

Abkar, M., and P. Moin, 2017: Large-eddy simulation of thermally stratified atmospheric boundary-layer flow using a minimum dissipation model. Bound.-Layer Meteor., 165, 405-419, https://doi.org/10.1007/s10546-017-0288-4.

— - H. J. Bae, and P. Moin, 2016: Minimum-dissipation scalar transport model for large-eddy simulation of turbulent flows. Phys. Rev. Fluids, 1, 041701, https://doi.org/10.1103/ PhysRevFluids.1.041701.

Alley, K. E., T. A. Scambos, M. R. Siegfried, and H. A. Fricker, 2016: Impacts of warm water on Antarctic ice shelf stability through basal channel formation. Nat. Geosci., 9, 290, https:// doi.org/10.1038/ngeo2675.

Arzeno, I. B., R. C. Beardsley, R. Limeburner, B. Owens, L. Padman, S. R. Springer, C. L. Stewart, and M. J. Williams, 2014: Ocean variability contributing to basal melt rate near the ice front of Ross Ice Shelf, Antarctica. J. Geophys. Res. Oceans, 119, 4214-4233, https://doi.org/10.1002/ 2014JC009792.

Begeman, C. B., and Coauthors, 2018: Ocean stratification and low melt rates at the Ross Ice Shelf grounding zone. J. Geophys. Res. Oceans, 123, 7438-7452, https://doi.org/10.1029/2018JC013987.

Bradshaw, P., and G. P. Huang, 1995: The law of the wall in turbulent flow. Proc. Roy. Soc. London, 451A, 165-188, https:// doi.org/10.1098/rspa.1995.0122.

Businger, J. A., J. C. Wyngaard, Y. Izumi, and E. F. Bradley, 1971: Flux-profile relationships in the atmospheric surface layer. J. Atmos. Sci., 28, 181-189, https://doi.org/10.1175/15200469(1971)028<0181:FPRITA $>2.0$. CO;2.

Dean, R., 1978: Reynolds number dependence of skin friction and other bulk flow variables in two-dimensional rectangular duct flow. J. Fluids Eng., 100, 215-223, https://doi.org/10.1115/ 1.3448633 .

Determann, J., and R. Gerdes, 1994: Melting and freezing beneath ice shelves: Implications from a three-dimensional oceancirculation model. Ann. Glaciol., 20, 413-419, https://doi.org/ 10.1017/S0260305500016785.

Deusebio, E., C. P. Caulfield, and J. R. Taylor, 2015: The intermittency boundary in stratified plane Couette flow. J. Fluid Mech., 781, 298-329, https://doi.org/10.1017/jfm.2015.497.

Eicken, H., H. Oerter, H. Miller, W. Graf, and J. Kipfstuhl, 1994: Textural characteristics and impurity content of meteoric and marine ice in the Ronne Ice Shelf, Antarctica. J. Glaciol., 40, 386-398, https://doi.org/10.1017/S0022143000007474.

Flores, O., and J. J. Riley, 2011: Analysis of turbulence collapse in the stably stratified surface layer using direct numerical simulation. Bound.-Layer Meteor., 139, 241-259, https://doi.org/ 10.1007/s10546-011-9588-2.

Foken, T., 2006: 50 years of the Monin-Obukhov similarity theory. Bound.-Layer Meteor., 119, 431-447, https://doi.org/10.1007/ s10546-006-9048-6. 
Gayen, B., R. W. Griffiths, and R. C. Kerr, 2016: Simulation of convection at a vertical ice face dissolving into saline water. J. Fluid Mech., 798, 284-298, https://doi.org/10.1017/ jfm.2016.315.

Gerland, S., J.-G. Winther, J. Børre Ørbæk, and B. V. Ivanov, 1999: Physical properties, spectral reflectance and thickness development of first year fast ice in Kongsfjorden, Svalbard. Polar Res., 18, 275-282, https://doi.org/10.1111/ j.1751-8369.1999.tb00304.x.

Grosfeld, K., R. Gerdes, and J. Determann, 1997: Thermohaline circulation and interaction between ice shelf cavities and the adjacent open ocean. J. Geophys. Res., 102, 15 595-15610, https://doi.org/10.1029/97JC00891.

Gudmundsson, G., 2013: Ice-shelf buttressing and the stability of marine ice sheets. Cryosphere, 7, 647-655, https://doi.org/ 10.5194/tc-7-647-2013.

Harig, C., and F. J. Simons, 2015: Accelerated West Antarctic ice mass loss continues to outpace East Antarctic gains. Earth Planet. Sci. Lett., 415, 134-141, https://doi.org/10.1016/ j.eps1.2015.01.029.

Hattermann, T., O. A. Nøst, J. M. Lilly, and L. H. Smedsrud, 2012: Two years of oceanic observations below the Fimbul Ice Shelf, Antarctica. Geophys. Res. Lett., 39, L12605, https://doi.org/ 10.1029/2012GL051012.

Holland, D. M., and A. Jenkins, 1999: Modeling thermodynamic iceocean interactions at the base of an ice shelf. J. Phys. Oceanogr., 29, 1787-1800, https://doi.org/10.1175/1520-0485(1999)029<1787: MTIOIA $>2.0 . \mathrm{CO} ; 2$.

Jacobs, S., C. Giulivi, and P. Mele, 2002: Freshening of the Ross Sea during the late 20th century. Science, 297, 386-389, https:// doi.org/10.1126/science.1069574.

Jenkins, A., 2011: Convection-driven melting near the grounding lines of ice shelves and tidewater glaciers. J. Phys. Oceanogr., 41, 2279-2294, https://doi.org/10.1175/JPO-D-11-03.1.

_, 2016: A simple model of the ice shelf-ocean boundary layer and current. J. Phys. Oceanogr., 46, 1785-1803, https://doi.org/ 10.1175/JPO-D-15-0194.1.

, and A. Bombosch, 1995: Modeling the effects of frazil ice crystals on the dynamics and thermodynamics of ice shelf water plumes. J. Geophys. Res., 100, 6967-6981, https:// doi.org/10.1029/94JC03227.

— , K. W. Nicholls, and H. F. J. Corr, 2010: Observation and parameterization of ablation at the base of Ronne Ice Shelf, Antarctica. J. Phys. Oceanogr., 40, 2298-2312, https://doi.org/ 10.1175/2010JPO4317.1.

Kader, B., and A. Yaglom, 1972: Heat and mass transfer laws for fully turbulent wall flows. Int. J. Heat Mass Transfer, 15, 23292351, https://doi.org/10.1016/0017-9310(72)90131-7.

Kaimal, J., J. Wyngaard, D. Haugen, O. Coté, Y. Izumi, S. Caughey, and C. Readings, 1976: Turbulence structure in the convective boundary layer. J. Atmos. Sci., 33, 2152-2169, https://doi.org/ 10.1175/1520-0469(1976)033<2152:TSITCB >2.0.CO;2.

Keitzl, T., J. P. Mellado, and D. Notz, 2016: Reconciling estimates of the ratio of heat and salt fluxes at the ice-ocean interface. J. Geophys. Res. Oceans, 121, 8419-8433, https://doi.org/ 10.1002/2016JC012018.

Kerr, R. C., and C. D. McConnochie, 2015: Dissolution of a vertical solid surface by turbulent compositional convection. J. Fluid Mech., 765, 211-228, https://doi.org/10.1017/jfm.2014.722.

Kimura, S., K. W. Nicholls, and E. Venables, 2015: Estimation of ice shelf melt rate in the presence of a thermohaline staircase. J. Phys. Oceanogr., 45, 133-148, https://doi.org/10.1175/JPOD-14-0106.1.
— A. Jenkins, P. Dutrieux, A. Forryan, A. C. Naveira Garabato, and Y. Firing, 2016: Ocean mixing beneath Pine Island glacier ice shelf, West Antarctica. J. Geophys. Res. Oceans, 121, 84968510, https://doi.org/10.1002/2016JC012149.

Mahrt, L., 2014: Stably stratified atmospheric boundary layers. Annu. Rev. Fluid Mech., 46, 23-45, https://doi.org/10.1146/ annurev-fluid-010313-141354.

Martin, S., and P. Kauffman, 1977: An experimental and theoretical study of the turbulent and laminar convection generated under a horizontal ice sheet floating on warm salty water. J. Phys. Oceanogr., 7, 272-283, https://doi.org/10.1175/15200485(1977)007<0272:AEATSO > 2.0.CO;2.

McConnochie, C., and R. Kerr, 2017: Testing a common ice-ocean parameterization with laboratory experiments. J. Geophys. Res. Oceans, 122, 5905-5915, https://doi.org/10.1002/2017JC012918.

_ , and — 2018: Dissolution of a sloping solid surface by turbulent compositional convection. J. Fluid Mech., 846, 563577, https://doi.org/10.1017/jfm.2018.282.

McPhee, M. G., 2008: Air-Ice-Ocean Interaction: Turbulent Ocean Boundary Layer Exchange Processes. Springer, 216 pp.

_ G. A. Maykut, and J. H. Morison, 1987: Dynamics and thermodynamics of the ice/upper ocean system in the marginal ice zone of the Greenland Sea. J. Geophys. Res., 92, 7017 7031, https://doi.org/10.1029/JC092iC07p07017.

_ J. Morison, and F. Nilsen, 2008: Revisiting heat and salt exchange at the ice-ocean interface: Ocean flux and modeling considerations. J. Geophys. Res., 113, C06014, https://doi.org/ 10.1029/2007JC004383.

Mondal, M., B. Gayen, R. W. Griffiths, and R. C. Kerr, 2019: Ablation of sloping ice faces into polar seawater. J. Fluid Mech., 863, 545-571, https://doi.org/10.1017/jfm.2018.970.

Monin, A. S., and A. M. Obukhov, 1954: Osnovnye zakonomernosti turbulentnogo peremesivanija $\mathrm{v}$ prizemnom sloe atmosfery (Basic laws of turbulent mixing in the surface layer of the atmosphere). Tr. Geofiz. Inst., Akad. Nauk SSSR, 24, 163-187.

Nicholls, K. W., S. Østerhus, K. Makinson, T. Gammelsrød, and E. Fahrbach, 2009: Ice-ocean processes over the continental shelf of the southern Weddell Sea, Antarctica: A review. Rev. Geophys., 47, RG3003, https://doi.org/10.1029/2007RG000250.

_ K. Kakinson, and E. Venables, 2012: Ocean circulation beneath Larsen C Ice Shelf, Antarctica from in situ observations. Geophys. Res. Lett., 39, L19608, https://doi.org/10.1029/ 2012 GL053187.

Obukhov, A. M., 1946: Turbulentnost'v temperaturnoj-neodnorodnoj atmosfere (turbulence in an atmosphere with a non-uniform temperature). Tr. Akad. Nauk. SSSR Inst. Teorel. Geofiz., 1, 95-115.

Oerter, H., J. Kipfstuhl, J. Determann, H. Miller, D. Wagenbach, A. Minikin, and W. Graft, 1992: Evidence for basal marine ice in the Filchner-Ronne Ice Shelf. Nature, 358, 399, https:// doi.org/10.1038/358399a0.

Orszag, S. A., 1971: Numerical simulation of incompressible flows within simple boundaries. I. Galerkin (spectral) representation. Stud. Appl. Math., 50, 293, https://doi.org/10.1002/sapm1971504293.

Pope, S. B., 2000: Turbulent Flows. Cambridge University Press, $806 \mathrm{pp}$.

Purkey, S. G., and G. C. Johnson, 2012: Global contraction of Antarctic Bottom Water between the 1980s and 2000s. J. Climate, 25, 5830-5844, https://doi.org/10.1175/JCLI-D-11-00612.1.

Rignot, E., and S. S. Jacobs, 2002: Rapid bottom melting widespread near Antarctic ice sheet grounding lines. Science, 296, 2020-2023, https://doi.org/10.1126/science.1070942. 
, and K. Steffen, 2008: Channelized bottom melting and stability of floating ice shelves. Geophys. Res. Lett., 35, L02503, https://doi.org/10.1029/2007GL031765.

Rozema, W., H. J. Bae, P. Moin, and R. Verstappen, 2015: Minimum-dissipation models for large-eddy simulation. Phys. Fluids, 27, 085107, https://doi.org/10.1063/1.4928700.

Rye, C. D., A. C. N. Garabato, P. R. Holland, M. P. Meredith, A. G. Nurser, C. W. Hughes, A. C. Coward, and D. J. Webb, 2014: Rapid sea-level rise along the Antarctic margins in response to increased glacial discharge. Nat. Geosci., 7, 732-735, https:// doi.org/10.1038/ngeo2230.

Schlichting, H., and K. Gersten, 2003: Boundary-Layer Theory. Springer, $800 \mathrm{pp}$.

Schoof, C., 2007: Ice sheet grounding line dynamics: Steady states, stability, and hysteresis. J. Geophys. Res., 112, F03S28, https:// doi.org/10.1029/2006JF000664.

Scotti, A., and B. White, 2016: The mixing efficiency of stratified turbulent boundary layers. J. Phys. Oceanogr., 46, 3181-3191, https://doi.org/10.1175/JPO-D-16-0095.1.

Sirevaag, A., 2009: Turbulent exchange coefficients for the ice/ ocean interface in case of rapid melting. Geophys. Res. Lett., 36, L04606, https://doi.org/10.1029/2008GL036587.

Snow, K., S. Rintoul, B. Sloyan, and A. M. Hogg, 2018: Change in dense shelf water and Adélie Land bottom water precipitated by iceberg calving. Geophys. Res. Lett., 45, 2380-2387, https:// doi.org/10.1002/2017GL076195.

Stanton, T. P., and Coauthors, 2013: Channelized ice melting in the ocean boundary layer beneath Pine Island Glacier. Science, 341, 1236-1239, https://doi.org/10.1126/science.1239373.
Taylor, J. R., 2008: Numerical simulations of the stratified oceanic bottom boundary layer. Ph.D. thesis, University of California, San Diego, 212 pp., https://escholarship.org/uc/item/5s30n2ts.

Vancoppenolle, M., C. M. Bitz, and T. Fichefet, 2007: Summer landfast sea ice desalination at Point Barrow, Alaska: Modeling and observations. J. Geophys. Res., 112, C04022, https:// doi.org/10.1029/2006JC003493.

Verstappen, R., 2016: How much eddy dissipation is needed to counterbalance the nonlinear production of small, unresolved scales in a large-eddy simulation of turbulence? Comput. Fluids, 176, 276-284, https://doi.org/10.1016/j.compfluid.2016.12.016.

Vreugdenhil, C. A., and J. R. Taylor, 2018: Large-eddy simulations of stratified plane Couette flow using the anisotropic minimum-dissipation model. Phys. Fluids, 30, 085104, https:// doi.org/10.1063/1.5037039.

Williams, M., R. Warner, and W. Budd, 1998: The effects of ocean warming on melting and ocean circulation under the Amery Ice Shelf, East Antarctica. Ann. Glaciol., 27, 75-80, https:// doi.org/10.3189/1998AoG27-1-75-80.

Wyngaard, J. C., 2010: Turbulence in the Atmosphere. Cambridge University Press, 406 pp.

Yaglom, A., and B. Kader, 1974: Heat and mass transfer between a rough wall and turbulent fluid flow at high Reynolds and Peclet numbers. J. Fluid Mech., 62, 601-623, https://doi.org/ 10.1017/S0022112074000838.

Zhou, Q., J. R. Taylor, and C. P. Caulfield, 2017: Self-similar mixing in stratified plane Couette flow for varying Prandtl number. J. Fluid Mech., 820, 86-120, https://doi.org/10.1017/ jfm.2017.200. 